\title{
Equilibrium of Surfaces in a Vertical Force Field
}

\author{
Antonio Martínez and A. L. Martínez-Triviño
}

\begin{abstract}
In this paper, we study $\varphi$-minimal surfaces in $\mathbb{R}^{3}$ when the function $\varphi$ is invariant under a two-parametric group of translations. Particularly those which are complete graphs over domains in $\mathbb{R}^{2}$. We describe a full classification of complete flat-embedded $\varphi$-minimal surfaces if $\varphi$ is strictly monotone and characterize rotational $\varphi$-minimal surfaces by its behavior at infinity when $\varphi$ has a quadratic growth.
\end{abstract}

Mathematics Subject Classification. 53C42, 35J60.

Keywords. $\varphi$-minimal, elliptic equation, weighted volume functional.

\section{Introduction}

The equilibrium of a flexible, inextensible surface $\Sigma$ in a force field $\mathcal{F}=$ $(X, Y, Z)$ of $\mathbb{R}^{3}$, was given by Poisson [20, pp. 173-187] and when the intrinsic forces of the surface are assumed to be equal, the external force must have a potential $\mathcal{T}$ which corresponds, up to a constant, with the tension of the surface, that is,

$$
\mathrm{d} \mathcal{T}+X \mathrm{~d} x+Y \mathrm{~d} y+Z \mathrm{~d} z=0 .
$$

In this case, the equilibrium condition is given in terms of the mean curvature vector $\mathbf{H}$ of $\Sigma$ as follows:

$$
\mathbf{H} \mathcal{T}+\mathcal{F}^{\perp}=0
$$

where $\perp$ denotes the projection to the normal bundle of $\Sigma$.

From equations (1.1) and (1.2), Poisson obtains:

- The minimal surface equation, by taking $\mathcal{F}=0$ and $\mathcal{T}=$ const.

- The capillary surface equation, by taking $\mathcal{T}=$ const and $\mathcal{F}$ normal to the surface with $\|\mathcal{F}\|$ depending linearly on the height.

The authors were partially supported by MICINN-FEDER, Grant No. MTM2016- 80313-P and Junta de Andalucía Grant No. FQM325. 
- The equation of a heavy surface in a gravitational field, by taking $\mathcal{T}=$ $(0,0, g \mathcal{E}(z)), g=$ gravitational constant and $\mathcal{E}(z)$ a density function on the surface.

In this paper, we are interested in the last case, that is, when the equation (1.2) gives

$$
\mathbf{H}=(\bar{\nabla} \varphi)^{\perp}=\dot{\varphi} \vec{e}_{3}^{\perp},
$$

where $\left.\varphi(z)=\log \int_{z_{0}}^{z} g \mathcal{E}(t)\right) \mathrm{d} t, \bar{\nabla}$ is the gradient operator in $\mathbb{R}^{3}$ and $\left({ }^{\cdot}\right)$ denotes derivate respect to the third coordinate. To get a regular problem, we have to restrict the surfaces to the region of $\mathbb{R}^{3}$ where $\varphi$ is regular. These surfaces are a particular case of the so called $f$-minimal surfaces (see [3]) for which the function $f$ depends only on the height. They can be viewed either as critical points of the weighted volume functional

$$
V_{\varphi}(\Sigma):=\int_{\Sigma} e^{\varphi} \mathrm{d} A_{\Sigma},
$$

where $\mathrm{d} A_{\Sigma}$ is the volume element of $\Sigma$, or as minimal surfaces in $\mathbb{R}^{3}$ with the conformally changed metric

$$
G_{\varphi}:=\mathrm{e}^{\varphi}\langle\cdot, \cdot\rangle
$$

From this property of minimality, a tangency principle can be applied and any two different $\varphi$-minimal surfaces cannot "touch" each other at one interior or boundary point (see [7, Theorem 1 and Theorem 1a]).

Any surface satisfying (1.3) will be called $\left[\varphi, \vec{e}_{3}\right]$-minimal and if $\Sigma$ is the vertical graph of a function $u: \Omega \subseteq \mathbb{R}^{2} \longrightarrow \mathbb{R}$, we also refer to $u$ as $\left[\varphi, \vec{e}_{3}\right]$ minimal. Hence, $u$ is $\left[\varphi, \vec{e}_{3}\right]$-minimal if and only if it solves the following $\left[\varphi, \vec{e}_{3}\right]$-minimal equation:

$$
\left(1+u_{x}^{2}\right) u_{y y}+\left(1+u_{y}^{2}\right) u_{x x}-2 u_{y} u_{x} u_{x y}=\dot{\varphi}(u)\left(1+u_{x}^{2}+u_{y}^{2}\right) .
$$

This kind of surfaces has been widely studied specially from the viewpoint of calculus of variations. Classical results about the Euler equation and the existence and regularity for the solutions of the Plateau problem for (1.4) can be found in $[2,9-11,24]$.

But contributions from a more geometric viewpoint only has been given for some particular functions $\varphi$. It is interesting to mention

- The case of $\varphi(z)=z$ : it corresponds with translating solitons, that is, surfaces in $\mathbb{R}^{3}$ such that

$$
t \mapsto \Sigma+t \vec{e}_{3}
$$

is a mean curvature flow, i.e., such that normal component of the velocity at each point is equal to the mean curvature at that point: $\mathbf{H}=\vec{e}_{3}^{\perp}$. Recent advances in the understanding of its local and global geometry can be found in $[4,8,12-14,16,17,23,25]$

- The case of $\varphi(z)=\alpha \log z, \alpha=$ const. It includes the two dimensional examples analogues of the catenaries (when $\alpha=1$ ). We refer to $[2,5,6$, $15,19]$ for some progress made in this family. 
The aim of this paper is develop a general and systematic approach to study $\left[\varphi, \vec{e}_{3}\right]$-minimal surfaces from a geometric viewpoint. Nonetheless, the class of $\left[\varphi, \vec{e}_{3}\right]$-minimal surfaces is indeed very large and much richer in what refers to examples and geometric behaviors. Although new ideas are needed for its study, it will be necessary, to get classification results, to impose some additional conditions to the function $\varphi$. Here, as a general assumption, we will always consider $\varphi$ strictly monotone, that is,

$$
\begin{aligned}
& \varphi:] a, b[\subseteq \mathbb{R} \rightarrow \mathbb{R} \text { is a strictly increasing (or decreasing) function } \\
& \text { and } \left.\Sigma \subset \mathbb{R}^{2} \times\right] a, b[\text {. }
\end{aligned}
$$

Invariant surfaces by an uniparametric group of rigid motions in $\mathbb{R}^{3}$ are related with the one-dimensional case of (1.6). Since $\varphi$ is taking so arbitrary, we only consider $\left[\varphi, \vec{e}_{3}\right]$-minimal surfaces invariant by two types of uniparametric groups, namely, groups of horizontal translations and the group of vertical rotations.

In the first case, besides vertical planes, we may consider that $u=u(x)$, $x \in I$ depends only on $x$. Then, from (1.6), the generalized cylinder $\Sigma=$ $\{(x, y, u(x)) \mid x \in I, y \in \mathbb{R}\}$ is a $\left[\varphi, \vec{e}_{3}\right]$-minimal surface if and only if $u$ satisfies

$$
u^{\prime \prime}(x)=\dot{\varphi}(u)\left(1+u^{\prime}(x)^{2}\right)
$$

From its physical interpretation, any solution of (1.8) will be called $\varphi$-catenary. The corresponding generalized cylinder is called $\left[\varphi, \vec{e}_{3}\right]$-catenary cylinder. If we rotate around the $x$-axis a $\left[\varphi, \vec{e}_{3}\right]$-catenary cylinder an angle $\theta \in] 0, \pi / 2\left[\right.$ and dilate by $\frac{1}{\cos \theta}$, the resulting surface is also $\left[\varphi, \vec{e}_{3}\right]$-minimal and we will say it is a tilted $\left[\varphi, \vec{e}_{3}\right]$-catenary cylinder. In Theorem 3.7 , we prove that any complete flat $\left[\varphi, \vec{e}_{3}\right]$-minimal surface is either a vertical plane or a $\left[\varphi, \vec{e}_{3}\right]$-catenary cylinder (maybe tilted).

In the second case, we consider $\left[\varphi, \vec{e}_{3}\right]$-minimal surfaces that are invariant under the one-parameter group of rotations that fix the $\vec{e}_{3}$ direction. From (1.6), the arc-length parametrized generating curve

$$
\gamma(s)=(x(s), 0, z(s)), \quad s \in I \subset \mathbb{R}
$$

of a such surface satisfies

$$
\left\{\begin{array}{l}
x^{\prime}=\cos (\theta) \\
z^{\prime}=\sin (\theta) \\
\theta^{\prime}=\dot{\varphi}(z) \cos (\theta)-\frac{\sin (\theta)}{x} .
\end{array}\right.
$$

In Theorems 4.5 and 4.11 , we establish the geometric properties of the rotational $\left[\varphi, \vec{e}_{3}\right]$-minimal surfaces according two types of surfaces: one is globally convex with only one complete embedded end (it is called a $\left[\varphi, \vec{e}_{3}\right]$-minimal bowl) and the other has two complete embedded convex ends and has a generating curve of winglike type (it is called $\left[\varphi, \vec{e}_{3}\right]$-minimal catenoid)

Very little is known about the geometry of the immersed $\left[\varphi, \vec{e}_{3}\right]$-minimal surfaces and most of the results have been proved only for translating solitons. One of the first result in that direction was obtained by Clutterbuck, Schnüre, Schulze in [4], where they proved that when $\dot{\varphi} \equiv 1$, any rotationally symmetric 
solution $u=u(r), r=\sqrt{x^{2}+y^{2}}$, on the exterior of a compact planar domain has de following asymptotic behavior:

$$
u(r)=\frac{r^{2}}{2}-\log r+O\left(r^{-1}\right) .
$$

Somewhat later Martin-Savas-Smoczyk proved in [17] that any complete translating soliton with a single end asymptotic to a translating paraboloid is a translating paraboloid.

In this paper, we generalize the above results to $\left[\varphi, \vec{e}_{3}\right]$-minimal with $\dot{\varphi}$ satisfying the following expansion at infinity:

$$
\dot{\varphi}(u)=\alpha u+\beta+\sum_{n=1}^{\infty} \frac{a_{n}}{u^{n}}, \quad a_{n} \in \mathbb{R},
$$

where either $\alpha>0$ and the first non-vanishing $a_{k}$ is positive or $\alpha=0$, $\beta>0$ and the first non-vanishing $a_{k}$ is negative. The results we prove can be summarized in the following two theorems

Theorem A. If $\dot{\varphi}$ satisfies (1.10), then any rotationally symmetric solution $u$ of (1.6) has the following asymptotic behavior:

- If $\alpha>0$,

$$
\varphi(u)(r)=C e^{\alpha r^{2}}+O\left(r^{2}\right), \quad C>0,
$$

- If $\alpha=0$ and up to a constant, we have

$$
\mathcal{G}(u)(r)=\frac{r^{2}}{2}-\frac{1}{\beta^{2}} \log (r)+O\left(r^{-2}\right),
$$

where $\mathcal{G}$ is the strictly increasing function given by $\mathcal{G}(u)=\int_{u_{0}}^{u} \frac{\mathrm{d} \xi}{\dot{\varphi}(\xi)}$.

Theorem B. Let $\Sigma$ be a complete properly embedded $\left[\varphi, \vec{e}_{3}\right]$-minimal surface in $\mathbb{R}^{3}$ with a single end that is smoothly asymptotic to a $\left[\varphi, \vec{e}_{3}\right]-$ minimal bowl, $\dot{\varphi}$ satisfying (1.10). Then, the surface $\Sigma$ is a $\left[\varphi, \vec{e}_{3}\right]$-minimal bowl.

The paper is organized as follows: in Sect. 2, we show some fundamental equations related to our family of surfaces and as a consequence, we prove the non-existence of closed examples and two results about strictly convexity and mean convexity of $\left[\varphi, \vec{e}_{3}\right]$-minimal surfaces.

Section 3 is devoted to the study and classification of embedded complete flat $\left[\varphi, \vec{e}_{3}\right]$-minimal surfaces. We describe geometrically the so called $\left[\varphi, \vec{e}_{3}\right]$-catenary cylinders and tilted $\left[\varphi, \vec{e}_{3}\right]$-catenary cylinders and characterize them together to vertical planes as the unique examples of complete flat $\left[\varphi, \vec{e}_{3}\right]$-minimal surfaces.

In Sect. 4, we study the existence and classification of rotational examples. We construct for $\varphi$ in a very general class of functions (strictly increasing and convex) a family of $\left[\varphi, \vec{e}_{3}\right]$-minimal bowls (which are strictly convex graphs) and $\left[\varphi, \vec{e}_{3}\right]$-minimal catenoids with a winglike shape (which resemble the usual translating catenoids in $\mathbb{R}^{3}$ ). 
Finally, Sects. 5 and 6 are devoted to study $\left[\varphi, \vec{e}_{3}\right]$-minimal surfaces when $\varphi$ has a quadratic growth. We provide the asymptotic behavior of rotationally symmetric examples and characterize $\left[\varphi, \vec{e}_{3}\right]$-minimal bowls by their behavior at infinity.

\section{Some Relevant Equations}

Here, we will give some local fundamental equations related to $\left[\varphi, \vec{e}_{3}\right]$-minimal surfaces. Let $\psi: M \longrightarrow \mathbb{R}^{3}$ be a 2-dimensional $\left[\varphi, \vec{e}_{3}\right]$-minimal immersion (maybe with a non empty boundary) with Gauss map $N$, induced metric $g$ and second fundamental form $\mathbf{A}$. We shall denote by $\nabla, \Delta$ and $\nabla^{2}$, respectively, the Gradient, Laplacian and Hessian operators of $g$.

The mean curvature vector of $\psi$ is defined by $\mathbf{H}=\operatorname{trace}_{g} \mathbf{A}$ and the symmetric bilinear form $\mathcal{A}$ given by $\mathcal{A}(X, Y)=-\langle\mathbf{A}(X, Y), N\rangle, X, Y \in T \Sigma$, is called scalar second fundamental form. The mean curvature function $H$ will be the trace of $\mathcal{A}$ with respect to $g$. With this notation, (1.3) is equivalent to

$$
H:=-\dot{\varphi}\left\langle N, \vec{e}_{3}\right\rangle \text {. }
$$

We will assume that $\varphi$ satisfies (1.7) and let us introduce the height and angle functions, respectively, by

$$
\mu:=\left\langle\psi, \vec{e}_{3}\right\rangle, \quad \eta:=\left\langle N, \vec{e}_{3}\right\rangle .
$$

Lemma 2.1. The following relations hold:

$$
\begin{aligned}
& \nabla \mu=\vec{e}_{3}^{\top}, \quad\langle\nabla \eta, \cdot\rangle=-\mathcal{A}(\nabla \mu, \cdot), \\
& \dot{\varphi}^{2}=\dot{\varphi}^{2}|\nabla \mu|^{2}+H^{2}, \\
& \dot{\varphi} \nabla^{2} \mu=H \mathcal{A}, \\
& \nabla^{2} \eta=(\nabla \mathcal{A})(\nabla \mu, \cdot, \cdot)+\frac{H}{\dot{\varphi}} \mathcal{A}^{[2]}, \\
& \Delta \mu=\dot{\varphi}\left(1-|\nabla \mu|^{2}\right), \\
& \Delta N+\dot{\varphi} \nabla \eta+\ddot{\varphi} \eta \nabla \mu+|\mathcal{A}|^{2} N=0, \\
& \nabla^{2} H=-\eta \nabla^{2} \dot{\varphi}-(\nabla \mathcal{A})(\nabla \varphi, \cdot, \cdot)-H \mathcal{A}^{[2]}+\mathcal{B} \\
& \Delta \mathcal{A}+(\nabla \mathcal{A})(\nabla \varphi, \cdot, \cdot)+\eta \nabla^{2} \dot{\varphi}+|\mathcal{A}|^{2} \mathcal{A}-\mathcal{B}=0,
\end{aligned}
$$

where $\mathcal{A}^{[2]}$ and $\mathcal{B}$ are the symmetric 2-tensors given by the following expressions:

$$
\begin{aligned}
& \mathcal{A}^{[2]}(X, Y)=\sum_{k} \mathcal{A}\left(X, E_{k}\right) \mathcal{A}\left(E_{k}, Y\right), \\
& \mathcal{B}(X, Y)=\langle\nabla \dot{\varphi}, X,\rangle \mathcal{A}(\nabla \mu, Y)+\langle\nabla \dot{\varphi}, Y\rangle \mathcal{A}(\nabla \mu, X),
\end{aligned}
$$

for any vector fields $X, Y \in T \Sigma$ and any orthonormal frame $\left\{E_{1}, E_{2}\right\}$ of $T \Sigma$.

Proof. (1) Differentiating $\mu$ and $\eta$ respect to any $X \in T \Sigma$, we get

$$
\begin{aligned}
& \langle\nabla \mu, X\rangle=d \mu(X)=\left\langle\vec{e}_{3}^{\top}, X\right\rangle, \\
& \langle\nabla \eta, X\rangle=\mathrm{d} \eta(X)=\left\langle d N(X), \vec{e}_{3}^{\top}\right\rangle=\mathcal{A}\left(X, \vec{e}_{3}^{\top}\right) .
\end{aligned}
$$


(2) From (2.1) and (1), it is clear that

$$
1=|\nabla \mu|^{2}+\frac{H^{2}}{\dot{\varphi}^{2}} .
$$

(3) From definition of the Hessian operator,

$\nabla^{2} \mu(X, Y)=X Y(\mu)-\left(\nabla_{X} Y\right)(\mu)=\left\langle\mathbf{A}(X, Y), e_{3}\right\rangle=-\mathcal{A}(X, Y) \eta$.

Therefore, (3) follows from (2.1).

(4) From Codazzi equation and (2.1):

$$
\begin{aligned}
& \nabla^{2} \eta(X, Y)=\sum_{k}(\nabla \mathcal{A})\left(E_{k}, X, Y\right) E_{k}(\mu)-\sum_{k} \mathcal{A}\left(X, E_{k}\right) \mathcal{A}\left(Y, E_{k}\right) \eta \\
& \quad=(\nabla \mathcal{A})(\nabla \mu, X, Y)+\frac{H}{\dot{\varphi}} A^{[2]}(X, Y) .
\end{aligned}
$$

(5) From (2) and (3),

$$
\Delta \mu=\sum_{k} \nabla^{2} \mu\left(E_{k}, E_{k}\right)=\frac{H^{2}}{\dot{\varphi}}=\dot{\varphi}\left(1-|\nabla \mu|^{2}\right) .
$$

(6) As $H=-\dot{\varphi} \eta$, we have

$$
\nabla H=-\ddot{\varphi} \eta \nabla u-\dot{\varphi} \nabla \eta,
$$

and (6) follows from the well-known fact that $\Delta N=\nabla H-|A|^{2} N$.

(7) From (2.1) and (4), we obtain

$$
\begin{aligned}
& \nabla^{2} H(X, Y)=X Y(H)-\left(D_{X} Y\right) H \\
& \quad=-\eta \nabla^{2} \dot{\varphi}(X, Y)-\dot{\varphi} \nabla^{2} \eta(X, Y)-\langle\nabla \dot{\varphi}, Y\rangle\langle X, \nabla \eta\rangle-\langle\nabla \dot{\varphi}, X\rangle\langle Y, \nabla \eta\rangle \\
& \quad=-\eta \nabla^{2} \dot{\varphi}(X, Y)-(\nabla \mathcal{A})(\nabla \varphi, X, Y)-H \mathcal{A}^{[2]}(X, Y)+\mathcal{B}(X, Y) .
\end{aligned}
$$

which give the proof of (7).

(8) Using the well-known Simon's identity:

$$
\Delta \mathcal{A}=\nabla^{2} H-|\mathcal{A}|^{2} \mathcal{A}+H \mathcal{A}^{[2]}
$$

and (7) we obtain (8).

From this Lemma, we have

Corollary 2.2. If $\varphi:] a, b[\rightarrow \mathbb{R}$, is a strictly increasing (or decreasing) function, then the height function $\mu$ of $\psi$ cannot attain a local maximum (or local minimum) at any interior point.

Corollary 2.3. There is no any closed 2-dimensional $\left[\varphi, \vec{e}_{3}\right]$-minimal immersion $\left.\psi: M \longrightarrow \mathbb{R}^{2} \times\right] a, b[$.

About the sign of the curvatures of $\psi$, we have

Theorem 2.4. Let $\varphi:] a, b[\rightarrow \mathbb{R}$ be a strictly increasing function satisfying

$$
\ddot{\varphi}+\lambda \dot{\varphi}^{2} \geq 0, \quad \text { for some constant } \lambda>0,
$$

and let $\left.\psi: \Sigma \longrightarrow \mathbb{R}^{2} \times\right] a, b\left[\right.$ be a 2-dimensional $\left[\varphi, \vec{e}_{3}\right]$-minimal immersion with $H \leq 0$. If $H$ vanishes anywhere, then $H$ vanishes everywhere and $\psi(\Sigma)$ lies in a vertical plane. 
Proof. Using (2.1) and the Eqs. (1), (2), (5) and (6) in Lemma 2.1, we have

$$
\begin{aligned}
& \Delta\left(\mathrm{e}^{-\lambda \varphi}\right)+\lambda \mathrm{e}^{-\lambda \varphi}\left(\ddot{\varphi}|\nabla \mu|^{2}+H^{2}-\lambda \dot{\varphi}^{2}|\nabla \mu|^{2}\right)=0, \\
& \Delta \eta+\dot{\varphi}\langle\nabla \eta, \nabla \mu\rangle+\left(|A|^{2}+\ddot{\varphi}|\nabla \mu|^{2}\right) \eta=0 .
\end{aligned}
$$

Thus, we obtain

$$
\begin{aligned}
& \Delta\left(\mathrm{e}^{-\lambda \varphi} \eta\right)+(2 \lambda+1)\left\langle\nabla\left(\mathrm{e}^{-\lambda \varphi} \eta\right), \nabla \varphi\right\rangle= \\
& \quad=-\eta \mathrm{e}^{-\lambda \varphi}\left((\lambda+1)\left(\ddot{\varphi}+\lambda \dot{\varphi}^{2}\right)|\nabla \mu|^{2}+\lambda H^{2}+|\mathcal{A}|^{2}\right) .
\end{aligned}
$$

But, by hypothesis, $\eta$ is a nonnegative function, and so, from the strong maximum principle, if it vanishes anywhere then it vanishes everywhere, which concludes the proof.

Theorem 2.5. Let $\varphi:] a, b[\rightarrow \mathbb{R}$ be a strictly increasing function satisfying $\dddot{\varphi} \leq 0$, and let $\left.\psi: \Sigma \longrightarrow \mathbb{R}^{2} \times\right] a, b\left[\right.$ be a 2 -dimensional locally convex $\left[\varphi, \vec{e}_{3}\right]$ minimal immersion. If the Gauss curvature $K$ vanishes anywhere, then $K$ vanishes everywhere.

Proof. By hypothesis, the Gauss map $N$ can be chosen such that $\mathcal{A}$ is a positive semi-definite bilinear form and from (8), we have

$$
\Delta \mathcal{A}+(\nabla \mathcal{A})(\nabla \varphi, ., .)+\mathcal{G}(\mathcal{A})=0
$$

where

$$
\mathcal{G}(\mathcal{A})=\eta \nabla^{2} \dot{\varphi}+|\mathcal{A}|^{2} \mathcal{A}-\mathcal{B} \text {. }
$$

But, from Lemma 2.1, if $\dddot{\varphi} \leq 0$, we obtain $\mathcal{G}(\mathcal{A})(v, v)=\eta \dddot{\varphi}\langle\nabla \mu, v\rangle^{2} \leq 0$ for each null vector $v$ of $\mathcal{A}$. Therefore, can apply the maximum principle of Hamilton (see [21, Section 2]) and if there is an interior point of $\Sigma$ where $\mathcal{A}$ has a null-eigenvalue then $\mathcal{A}$ must have a null-eigenvalue everywhere, which concludes the proof of the theorem.

\section{Complete Flat $\left[\varphi, \vec{e}_{3}\right]$-Minimal Surfaces}

\subsection{Vertical Graphs Invariant by Horizontal Translations}

Consider the $\left[\varphi, \vec{e}_{3}\right]$-minimal vertical graph given by a function $u$ which only depend on one variable, $u=u(x)$, from (1.6) $u$ must be a solution of the following ODE:

$$
u^{\prime \prime}(x)=\dot{\varphi}(u)\left(1+u^{\prime}(x)^{2}\right)
$$

To look for complete examples, we will consider that

$$
\varphi:] a, \infty[\longrightarrow \mathbb{R}
$$

is either a strictly increasing (or decreasing) function. Then, by taking $z=$ $\varphi(u)$ and $u^{\prime}=\tan (v)$, we obtain that (3.1) is equivalent to

$$
\left.\begin{array}{l}
v^{\prime}=h(z), \\
z^{\prime}=h(z) \tan (v),
\end{array}\right\}
$$

where $h(z)=\dot{\varphi}\left(\varphi^{-1}(z)\right)$. 


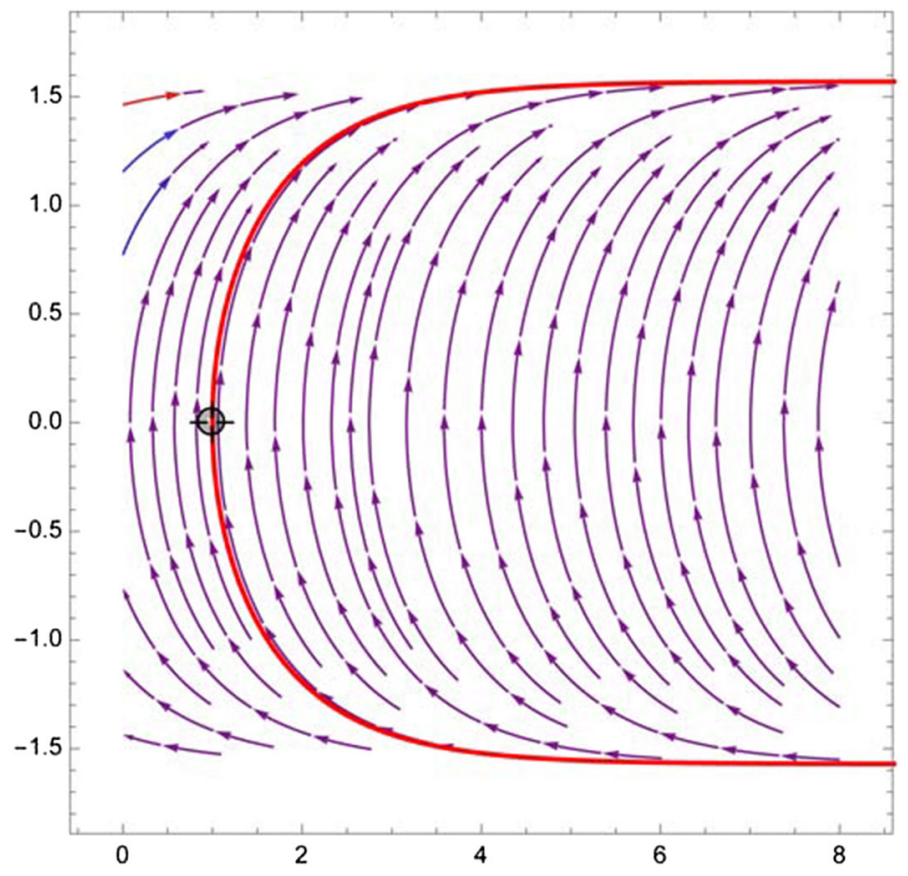

Figure 1. Phase portrait of (3.2)

It is clear that $e^{z} \cos (v)$ is constant along the solutions of (3.2) and from Fig. 1, for each solution $u$ of (3.1) there exists a unique $x_{0} \in \mathbb{R}$ such that $v\left(x_{0}\right)=0$ (it is not a restriction to assume that $x_{0}=0$ ).

By taking the initial conditions

$$
u(0)=u_{0}, \quad u^{\prime}(0)=0,
$$

we have that for each $x \geq 0, u(x)$ is given by

$$
u(x):=(\mathcal{X} \circ \varphi)^{-1}(x), \quad \text { with } \quad \mathcal{X}(z)=\int_{z_{0}}^{z} \frac{\mathrm{d} \tau}{|h(\tau)| \sqrt{\mathrm{e}^{2\left(\tau-z_{0}\right)}-1}},
$$

where $z_{0}=\varphi\left(u_{0}\right)$. Thus, from (3.1) and (3.3), we obtain,

Proposition 3.1. The solution $u$ of (3.1)-(3.3) is even and it is defined in the interval $]-\Lambda_{u_{0}}, \Lambda_{u_{0}}[$, where

$$
\Lambda_{u_{0}}=\lim _{u \rightarrow \infty} \int_{\varphi\left(u_{0}\right)}^{\varphi(u)} \frac{\mathrm{d} \tau}{|h(\tau)| \sqrt{\mathrm{e}^{2\left(\tau-z_{0}\right)}-1}} .
$$

Theorem 3.2. If $\varphi:] a, \infty[\longrightarrow \mathbb{R}$ is a strictly increasing function, then,

- $\Lambda_{u_{0}}<\infty$ if and only if $\int_{u_{0}}^{\infty} \mathrm{e}^{-\varphi(\lambda)} \mathrm{d} \lambda<\infty$. Therefore, if $\Lambda_{\lambda_{0}}<\infty$ for some $\left.\lambda_{0} \in\right] a, \infty\left[\right.$, then $\Lambda_{\lambda}<\infty$ for all $\left.\lambda \in\right] a, \infty[$.

- If $\Lambda_{\lambda}<\infty$ and $\dot{\varphi}$ is increasing (respectively, decreasing), then $\Lambda_{\lambda}$ is decreasing (respectively, increasing) in $\lambda$. 
Proof. As

$$
\lim _{\tau \rightarrow \infty} \frac{\sqrt{\mathrm{e}^{2\left(\tau-z_{0}\right)}-1}}{\mathrm{e}^{\tau-z_{0}}}=1 \neq 0,
$$

the first item follows from (3.5).

On the other hand, by assuming that $\dot{\varphi}$ is increasing and $\Lambda_{\lambda}<\infty$ for all $\lambda \in] a, \infty\left[\right.$, we have from (3.5), that, if $\lambda_{1} \leq \lambda_{2}$,

$$
\Lambda_{\lambda_{1}} \geq \Lambda_{\lambda_{2}}+\lim _{z \rightarrow \infty} \int_{z-\varphi\left(\lambda_{2}\right)}^{z-\varphi\left(\lambda_{1}\right)} \frac{\mathrm{d} \tau}{h\left(\tau+\varphi\left(\lambda_{1}\right)\right) \sqrt{\mathrm{e}^{2 \tau}-1}}=\Lambda_{\lambda_{2}} .
$$

A similar discussion can be done when $\dot{\varphi}$ is decreasing.

From (3.1), (3.2), (3.3), (3.4), (3.5) and Theorem 3.2, we can prove the following properties of the solutions,

Theorem 3.3. Let $\varphi:] a, \infty[\longrightarrow] b, c[, a, b \in \mathbb{R} \cup\{-\infty\}, c \in \mathbb{R} \cup\{\infty\}$ be a strictly increasing diffeomorphism, then the solution $u$ of (3.1)-(3.3) is defined in $]-\Lambda_{u_{0}}, \Lambda_{u_{0}}\left[, \Lambda_{u_{0}} \in\left\{\mathbb{R}^{+}, \infty\right\}\right.$, it is convex, symmetric about the $y$-axis and has a minimum at $x=0$. Moreover,

- if $c<\infty$, then $\Lambda_{u_{0}}=\infty$, and

$$
\lim _{x \rightarrow \pm \infty} u(x)=\infty, \quad \lim _{x \rightarrow \pm \infty} u^{\prime}(x)= \pm \sqrt{\mathrm{e}^{2\left(c-z_{0}\right)}-1} .
$$

- if $c=\infty$,

$$
\lim _{x \rightarrow \pm \Lambda_{u_{0}}} u(x)=\infty, \quad \lim _{x \rightarrow \pm \Lambda_{u_{0}}} u^{\prime}(x)= \pm \infty .
$$

In particular, if $\Lambda_{u_{0}}<\infty$, the graph of $u$ is asymptotic to two vertical lines.

Theorem 3.4. Let $\varphi:] a, \infty[\longrightarrow] b, c[, a, b \in\{\mathbb{R},-\infty\}, c \in\{\mathbb{R}, \infty\}$ be $a$ strictly decreasing diffeomorphism, then the solution $u$ of (3.1)-(3.3) is defined in $]-\Lambda_{u_{0}}, \Lambda_{u_{0}}\left[, \Lambda_{u_{0}} \in\left\{\mathbb{R}^{+}, \infty\right\}\right.$, it is concave, symmetric about the $y$-axis and has a maximum at $x=0$. Moreover,

- if $c<\infty$, then $\Lambda_{u_{0}}<\infty$, and

$$
\lim _{x \rightarrow \pm \Lambda_{u_{0}}} u(x)=a, \quad \lim _{x \rightarrow \pm \Lambda_{u_{0}}} u^{\prime}(x)= \pm \sqrt{\mathrm{e}^{2\left(c-z_{0}\right)}-1} .
$$

- if $c=\infty$, then

$$
\Lambda_{u_{0}}<\infty \Longleftrightarrow \int_{a}^{u_{0}} \mathrm{e}^{-\varphi(\lambda)} \mathrm{d} \lambda<\infty
$$

and

$$
\lim _{x \rightarrow \pm \Lambda_{u_{0}}} u(x)=a, \quad \lim _{x \rightarrow \pm \Lambda_{u_{0}}} u^{\prime}(x)= \pm \infty
$$

Remark 3.5. In the hypothesis of Theorem 3.4, the graph of $u$ is complete when $a=-\infty$. But in this case, by changing $\varphi$ by $-\varphi$, we can also apply Theorem 3.3.

Definition 3.6. For each solution $u$ of $(3.1)-(3.3)$, we refer $\mathcal{C}:=\operatorname{Graph}(u) \times \mathbb{R}$ as a $\left[\varphi, \vec{e}_{3}\right]$-catenary cylinder surface (Fig. 2). 

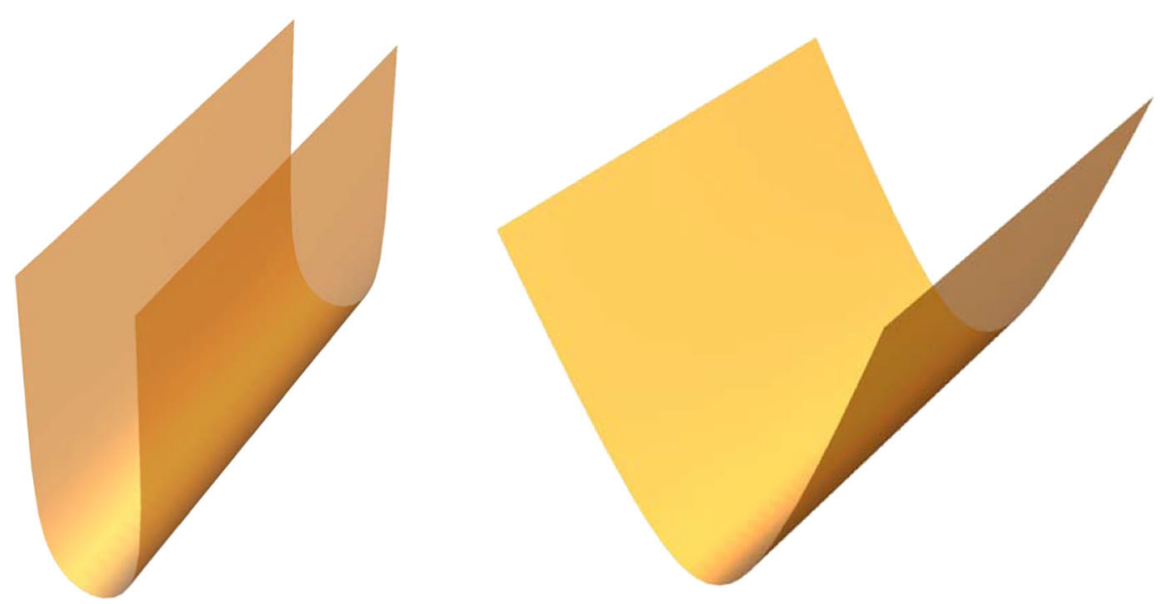

Figure 2. $\left[\varphi, \vec{e}_{3}\right]$-catenary cylinders with $\dot{\varphi}=1$ and $\dot{\varphi}=$ $1 / u^{2}$, respectively

\subsection{Tilted $\left[\varphi, \vec{e}_{3}\right]$-Catenary Cylinders}

Let $\psi:=(x, y, u(x)), x \in]-\Lambda_{u_{0}}, \Lambda_{u_{0}}\left[\right.$ be a $\left[\varphi, \vec{e}_{3}\right]$-catenary cylinder with $u$ satisfying (3.3) and Gauss map,

$$
N=\frac{1}{\sqrt{1+u^{\prime 2}}}\left(u^{\prime}, 0,-1\right) .
$$

If we rotate the surface by an angle $\theta \in] 0, \pi / 2[$ about the $x$-axis and dilate by $1 / \cos \theta$, the resulting surface may be written as follows:

$$
\widetilde{\psi}=\psi+\frac{1-\cos \theta}{\cos \theta}\left\langle\psi, \vec{e}_{1}\right\rangle \vec{e}_{1}+(\tan \theta) \vec{e}_{1} \wedge \psi
$$

where $\vec{e}_{1}=(1,0,0)$ and whose Gauss map is given by

$$
\widetilde{N}=\cos \theta N+(1-\cos \theta)\left\langle N, \vec{e}_{1}\right\rangle \vec{e}_{1}+\sin \theta \vec{e}_{1} \wedge N
$$

The mean curvature $\widetilde{H}$ of $\widetilde{\psi}$ verifies

$$
\widetilde{H}=\cos \theta H=-\cos \theta \dot{\varphi}\left\langle\vec{e}_{3}, N\right\rangle=-\dot{\varphi}\left\langle\vec{e}_{3}, \widetilde{N}\right\rangle .
$$

Consequently, $\widetilde{\psi}$ is also $\left[\varphi, \vec{e}_{3}\right]$-minimal and we are going to refer these examples as tilted $\left[\varphi, \vec{e}_{3}\right]$-catenary cylinders.

Observe that,

$$
\widetilde{\psi}(x, y):=\left(\frac{x}{\cos \theta}, y-u(x) \tan \theta, u(x)+y \tan \theta\right),
$$

and it is the graph of the function

$$
\begin{aligned}
& \left.\mathcal{C}_{\theta}:\right]-\frac{\Lambda_{u_{0}}}{\cos \theta}, \frac{\Lambda_{u_{0}}}{\cos \theta}[\times \mathbb{R} \longrightarrow \mathbb{R} \\
& \mathcal{C}_{\theta}(x, y)=\frac{u(x \cos \theta)}{\cos ^{2} \theta}+y \tan \theta
\end{aligned}
$$



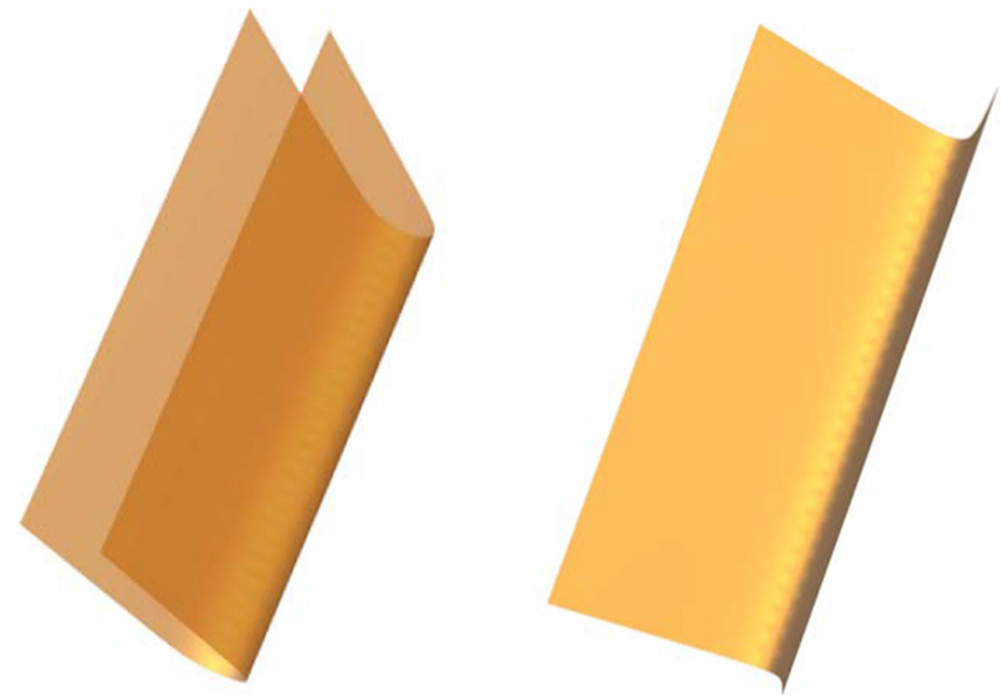

Figure 3. Titled $\left[\varphi, \vec{e}_{3}\right]$-catenary cylinders with $\dot{\varphi}=1$ and $\dot{\varphi}=1 / u^{3}$, respectively

Theorem 3.7. Let $\Sigma \subset \mathbb{R}^{3}$ be a complete flat $\left[\varphi, \vec{e}_{3}\right]$-minimal surface. If $\varphi$ : $\mathbb{R} \rightarrow \mathbb{R}$ is a strictly increasing diffeomorphism, then $\Sigma$ is either a vertical plane or a $\left[\varphi, \vec{e}_{3}\right]$-catenary cylinder (maybe tilted) surface (Fig. 3).

Proof. From basic differential geometry, $\Sigma=\alpha \times \Pi^{\perp}$ is a ruled surface and its Gauss map is constant along the rules, where $\alpha$ is a complete regular curve in a plane $\Pi \subset \mathbb{R}^{3}$.

Claim Let $\mathcal{L}$ be a straight line of $\Sigma$ and $\mathcal{V}_{L}$ be the unit normal vector along $\mathcal{L}$. If $\left\langle\mathcal{V}_{L}, \vec{e}_{3}\right\rangle \neq 0$, then there exists a $\left[\varphi, \vec{e}_{3}\right]$-catenary cylinder $\mathcal{C}_{\mathcal{L}}$ (tilted, if $\mathcal{L}$ is not horizontal) containing $\mathcal{L}$ and tangent to $\Sigma$ along $\mathcal{L}$.

Then, up to an appropriate rotation and dilatation, $\Sigma$ is tangent to a $\left[\varphi, \vec{e}_{3}\right]$ catenary cylinder along a rule. The result follows from standard theory of uniqueness of solution for the ODE (3.1).

Proof of the Claim. If $\mathcal{L}$ is horizontal then, after a rotation about the axis $\vec{e}_{3}$, we may assume that

$$
\mathcal{L}=\left\{\left(x_{0}, 0, u_{0}\right)+s(0,1,0) \mid s \in \mathbb{R}\right\}
$$

and there exists $\phi \in]-\pi / 2, \pi / 2\left[\right.$ such that $\mathcal{V}_{\mathcal{L}}=(-\sin \phi, 0, \cos \phi)$. Then, as $\varphi: \mathbb{R} \rightarrow \mathbb{R}$ is a strictly increasing diffeomorphism, from (3.1), there exists a solution $u_{\mathcal{L}}$ of $(3.1)-(3.3)$ and $x_{1} \in \mathbb{R}$, such that $u_{\mathcal{L}}\left(x_{1}\right)=u_{0}$ and $u_{\mathcal{L}}^{\prime}\left(x_{1}\right)=$ $\tan \phi$. The $\left[\varphi, \vec{e}_{3}\right]$-catenary cylinder we are looking for is just a translation in the $\vec{e}_{1}$-axis of the catenary cylinder $\mathcal{C}_{u_{\mathcal{L}}}$ associated to $u_{\mathcal{L}}$. 
If $\mathcal{L}$ is not horizontal and $p=\mathcal{L} \cap\{z=0\}$, then by rotation of center $p$ and axis $\vec{e}_{3}$ we may assume there exists $\left.\theta \in\right]-\pi / 2,0[$ and $\alpha \in \mathbb{R}$, such that

$$
\mathcal{V}_{\mathcal{L}}=\frac{1}{\sqrt{\alpha^{2}+1}}(-\alpha,-\sin \theta, \cos \theta)
$$

Therefore, from (3.6) and (3.7), if we take the solution $u_{\mathcal{L}}$ of $(3.1)-(3.3)$ satisfying

$$
u_{\mathcal{L}}\left(x_{1}\right)=\left\langle p, \vec{e}_{2}\right\rangle \cos \theta \sin \theta, \quad u_{\mathcal{L}}^{\prime}\left(x_{1}\right)=\alpha,
$$

for some $x_{1} \in \mathbb{R}$, we conclude that our tilted $\left[\varphi, \vec{e}_{3}\right]$-catenary cylinder is a translation in the $\vec{e}_{1}$-axis of the tilted $\left[\varphi, \vec{e}_{3}\right]$-catenary cylinder obtained after rotation of angle $\theta$ around the $\vec{e}_{2}$-axis and dilation of $1 / \cos \theta$ the $\left[\varphi, \vec{e}_{3}\right]$ catenary cylinder associated to $u_{\mathcal{L}}$.

As consequence, from the Theorem 2.5, the following result holds:

Corollary 3.8. Let $\varphi:] a, b[\rightarrow \mathbb{R}$ be a strictly increasing function satisfying $\dddot{\varphi} \leq 0$, and let $\Sigma$ be a complete locally convex $\left[\varphi, \vec{e}_{3}\right]$-minimal immersion in $\left.\mathbb{R}^{2} \times\right] a, b[$. If the Gauss curvature $K$ vanishes anywhere, then $\Sigma$ is either $a$ vertical plane or a $\left[\varphi, \vec{e}_{3}\right]$-catenary cylinder (maybe tilted) surface.

\section{4. $\left[\varphi, \vec{e}_{3}\right]$-Minimal Surfaces of Revolution}

In this section, we are going to study geometric behavior of rotationally symmetric solutions of (1.6).

\subsection{The Singular Case}

In the rotationally symmetric case, the Eq. (1.6) reduces to the following ordinary differential equation for $u=u(r), r=\sqrt{x^{2}+y^{2}}$ :

$$
u^{\prime \prime}=\left(1+u^{\prime 2}\right)\left(\dot{\varphi}(u)-\frac{u^{\prime}}{r}\right) \text {, }
$$

where (') denotes derivative respect to $r$ and $\varphi:] a, b[\subseteq \mathbb{R} \longrightarrow \mathbb{R}$ is a smooth function. Since (4.1) is degenerated, the existence and uniqueness of solution at $r=0$ is not assured by standard theory. Multiplying by $r$ we obtain that (4.1) also writes as

$$
\left(\frac{r u^{\prime}}{\sqrt{1+u^{\prime 2}}}\right)^{\prime}=\frac{r \dot{\varphi}(u)}{\sqrt{1+u^{\prime 2}}} .
$$

But, from [22, Theorem 2], a solution of (1.6) cannot possess isolated nonremovable singularities, hence, it is not a restriction to look for the existence of solutions of (4.2) with the following initial conditions:

$$
\left.u(0)=u_{0} \in\right] a, b\left[, \quad u^{\prime}(0)=0 .\right.
$$

In this sense and using a similar argument to [15, Proposition 2], we can assert 
Proposition 4.1. The problem (4.1)-(4.3) has a unique solution $u \in \mathcal{C}^{2}([0, R])$ for some $R>0$ which depends continuously on the initial data and such that

$$
u^{\prime \prime}(0)=\frac{\dot{\varphi}\left(u_{0}\right)}{2} .
$$

The following result allows us to compare rotational symmetric $\left[\varphi, \vec{e}_{3}\right]$ minimal graphs:

Proposition 4.2. Let $\left.\varphi_{1}, \varphi_{2}:\right] a, b[\rightarrow \mathbb{R}$ be strictly increasing and convex functions satisfying that $\dot{\varphi}_{1}>\dot{\varphi}_{2}$ on $] a, b\left[\right.$ and denote by $u_{\varphi_{1}}$ and $u_{\varphi_{2}}$ the $\left[\varphi_{i}, \vec{e}_{3}\right]-$ minimal graphs solutions to the corresponding problem (4.1)-(4.3). Then

$$
\left.u_{\varphi_{1}}^{\prime}>u_{\varphi_{2}}^{\prime}, \quad \text { on }\right] 0, r_{0}[\text {. }
$$

Proof. If we take the function $d:=u_{\varphi_{1}}^{\prime}-u_{\varphi_{2}}^{\prime}$, then $d(0)=0$ and

$$
d^{\prime}(0)=u_{\varphi_{1}}^{\prime \prime}(0)-u_{\varphi_{2}}^{\prime \prime}(0)=\left(\frac{\dot{\varphi}_{1}\left(u_{0}\right)}{2}-\frac{\dot{\varphi}_{2}\left(u_{0}\right)}{2}\right)>0 .
$$

Hence, there exists $\epsilon>0$ such that $d=u_{\varphi_{1}}^{\prime}-u_{\varphi_{2}}^{\prime}>0$ on $] 0, \epsilon[$. If there exists $r_{1}>0$ satisfying $d\left(r_{1}\right) \leq 0$, we can take $r^{*}:=\inf \{r>0: d(r)<0\}$ so that $d\left(r^{*}\right)=0$ and $d^{\prime}\left(r^{*}\right) \leq 0$. But, from (4.1) and having in mind that $\int_{0}^{r^{*}} d>0$, we get

$$
\begin{aligned}
0 \geq d^{\prime}\left(r^{*}\right) & =\left(1+u_{\varphi_{1}}^{\prime}\left(r^{*}\right)^{2}\right)\left[\dot{\varphi}_{1}\left(u_{\varphi_{1}}\left(r^{*}\right)\right)-\dot{\varphi}_{2}\left(u_{\varphi_{2}}\left(r^{*}\right)\right]\right. \\
& >\left(1+u_{\varphi_{1}}^{\prime}\left(r^{*}\right)^{2}\right)\left[\dot{\varphi}_{1}\left(u_{\varphi_{2}}\left(r^{*}\right)\right)-\dot{\varphi}_{2}\left(u_{\varphi_{2}}\left(r^{*}\right)\right]>0,\right.
\end{aligned}
$$

which is a contradiction.

Remark 4.3. The above Proposition also holds if we assume that $\varphi_{1}, \varphi_{2}$ : ]$a, b[\rightarrow \mathbb{R}$ are smooth functions so that

$$
\left.\inf \dot{\varphi}_{1}>\sup \dot{\varphi}_{2}, \quad \text { on }\right] a, b[\text {. }
$$

As consequence of Proposition 4.2 and the asymptotic behavior of rotational solitons proved in [4], we have

Corollary 4.4. Let $\varphi:[a,+\infty[\rightarrow \mathbb{R}$ be strictly increasing regular function and $u$ be an entire solution of (4.1). If there exists $\alpha>0$ such that $\dot{\varphi}>\alpha$, then

$$
u^{\prime}(r) \geq \alpha r-\frac{1}{\alpha r},
$$

for $r$ large enough.

\subsection{Geometric Description of Revolution $\left[\varphi, \vec{e}_{3}\right]$-Minimal Surfaces}

Now, we want to describe $\left[\varphi, \vec{e}_{3}\right]$-minimal surfaces that are invariant under the one-parameter group of rotations that fix the $\vec{e}_{3}$ direction. A such surface with generating curve the arc-length parametrized curve

$$
\gamma(s)=(x(s), 0, z(s)), \quad s \in I \subset \mathbb{R}
$$

is given by

$$
\psi(s, t)=(x(s) \cos (t), x(s) \sin (t), z(s)),(s, t) \in I \times \mathbb{R} .
$$


The inner normal of $\psi$ writes as

$$
N(s, t)=\left(-z^{\prime}(s) \cos (t),-z^{\prime}(s) \sin (t), x^{\prime}(s)\right),
$$

and the coefficients of the first and second fundamental form,

$$
\begin{aligned}
& \left\langle\psi_{s}, \psi_{s}\right\rangle=1,\left\langle\psi_{s}, N_{s}\right\rangle=-\kappa, \\
& \left\langle\psi_{t}, \psi_{t}\right\rangle=x^{2},\left\langle\psi_{t}, N_{t}\right\rangle=-x z^{\prime}, \\
& \left\langle\psi_{s}, \psi_{t}\right\rangle=0, \quad\left\langle\psi_{s}, N_{t}\right\rangle=0,
\end{aligned}
$$

where $\kappa$ is the curvature of $\gamma$ and by ' we denote derivative respect to $s$.

From (4.6), the mean curvature vector of $\psi$ is given by

$$
\mathbf{H}=-\left(\kappa+\frac{z^{\prime}}{x}\right) N .
$$

Consequently, from $(2.1),(4.4)$ and $(4.5)$, the surface $\psi$ is a $\left[\varphi, \vec{e}_{3}\right]$-minimal surface if and only if

$$
\left\{\begin{array}{l}
x^{\prime}=\cos (\theta) \\
z^{\prime}=\sin (\theta) \\
\theta^{\prime}=\dot{\varphi}(z) \cos (\theta)-\frac{\sin (\theta)}{x}
\end{array}\right.
$$

where $\theta(s)=\int_{0}^{s} \kappa(t) \mathrm{d} t$.

Along this section, we will consider that $\varphi:] a, \infty[\longrightarrow \mathbb{R}$ is a strictly increasing and convex function, that is

$$
\dot{\varphi}>0, \quad \ddot{\varphi} \geq 0, \quad \text { on }] a, \infty[.
$$

4.2.1. Globally Convex Examples. Here, we want to study the solutions of (4.8) with the following initial conditions,

$$
\left.x(0)=0, \quad z(0)=z_{0} \in\right] a, \infty[, \quad \theta(0)=0 .
$$

In this case, the surface intersects orthogonally the rotation axis and we have the following result:

Theorem 4.5. If $x_{0}=0$, then $\gamma$ is the graph of a strictly convex symmetric function $u(x)$ defined on a maximal interval $]-\omega_{+}, \omega_{+}[$which has a minimum at 0 and

$$
\lim _{x \rightarrow \pm \omega_{+}} u(x)=\infty
$$

Proof. First of all, we remark that the existence of $\gamma$ around $s=0$ is guaranteed from Proposition 4.1.

Moreover, it is easy to see that $\bar{x}(s)=-x(-s), \bar{z}(s)=z(-s)$ and $\bar{\theta}(s)=-\theta(-s)$ are also solutions of the same initial value problem (4.8)(4.10). Hence, $\gamma$ is symmetric respect to $\vec{e}_{3}$ direction and we may consider only the case $s \geq 0$.

By application of L'Hôpital's rule, we have that $2 \theta^{\prime}(0)=\dot{\varphi}\left(z_{0}\right)>0$ and $\gamma$ is a strictly locally convex planar curve around of $s=0$. We assert that $\theta^{\prime}(s)>0$ for $s \geq 0$, otherwise from (4.10), there exists a first value $s_{0}>0$ 

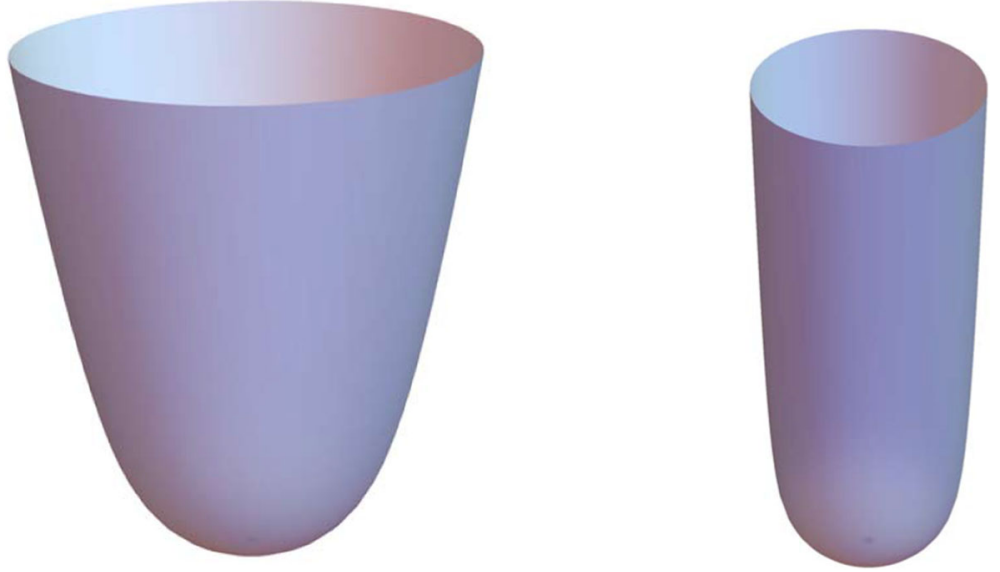

Figure 4. $\left[\varphi, \vec{e}_{3}\right]$-minimal bowls for $\dot{\varphi}(u)=e^{-1 / u}($ left $)$ and $\dot{\varphi}(u)=u^{2}$ (right)

such that $\theta^{\prime}\left(s_{0}\right)=0$ and $\theta^{\prime \prime}\left(s_{0}\right) \leq 0$. As $\theta^{\prime}>0$ on $\left[0, s_{0}[\right.$, from (4.8) we have that $0<2 \theta\left(s_{0}\right)<\pi$ and by differentiation of (4.8), we get

$$
\theta^{\prime \prime}\left(s_{0}\right)=\frac{\sin \left(2 \theta\left(s_{0}\right)\right)}{2}\left(\ddot{\varphi}\left(z\left(s_{0}\right)\right)+\frac{1}{x\left(s_{0}\right)^{2}}\right)>0,
$$

getting a contradiction.

In the same way, as $\theta^{\prime}>0$ for $s>0$, we have that $0<2 \theta(s)<\pi$ for $s>0$ and $\gamma$ is the graph of a strictly convex function $u=u(x)$ which is a $\mathcal{C}^{2}$ solution of

$$
\begin{cases}u^{\prime \prime}=\left(1+u^{\prime 2}\right) & \left(\dot{\varphi}(u)-\frac{u^{\prime}}{x}\right)>0, \\ u(0)=z_{0}, & u^{\prime}(0)=0,\end{cases}
$$

on the maximal interval of existence $]-\omega_{+}, \omega_{+}\left[\right.$. Finally, if $\lim _{x \rightarrow \pm \omega_{+}} u(x)=$ $h_{0}<\infty$, then the standard theory of prolongation of solutions, gives that $\omega_{+}=+\infty$ which is also a contradiction by the convexity of $u$ (Fig. 4).

Definition 4.6. If $\gamma$ is a graph as in Theorem 4.5, we are going to say that the revolution surface with generating curve $\gamma$ is a $\left[\varphi, \vec{e}_{3}\right]$-minimal bowl.

4.2.2. Non-convex Examples. Now, we want to study the solutions of (4.8) with the following initial conditions:

$$
\left.x(0)=x_{0}>0, \quad z(0)=z_{0} \in\right] a, \infty[, \quad \theta(0)=0 .
$$

From standard theory, the existence and uniqueness of solution to the problem (4.8)-(4.13) is guaranteed.

Let ] $-s_{-}, s_{+}$[ be the maximal interval of existence and consider $\gamma^{+}:=$ $\left.\gamma\right|_{\left[0, s_{+}[\right.}$the right branch of $\gamma$. Arguing as in Theorem 4.5, we can prove that $\gamma^{+}$is the graph of a convex function $u=u(x)$ defined on a maximal interval 
]$x_{0}, \omega_{+}[$, such that

$$
\lim _{x \rightarrow \omega_{+}} u(x)=\infty .
$$

For studying the left branch of $\gamma$, we are going to consider, $\gamma^{-}(s)=$ $\gamma(-s)$ for $s \in\left[0, s_{-}[\right.$. Then, by taking $\bar{x}(s)=x(-s), \bar{z}(s)=z(-s)$ and $\bar{\theta}(s)=\theta(-s)+\pi$ for $s \in\left[0, s_{-}[\right.$, we have that $\{\bar{x}, \bar{z}, \bar{\theta}\}$ is a solution of $(4.8)$ on $\left[0, s_{-}[\right.$satisfying

$$
\left.\bar{x}(0)=x_{0}>0, \quad \bar{z}(0)=z_{0} \in\right] a, \infty[, \quad \bar{\theta}(0)=\pi .
$$

Lemma 4.7. There exists $\left.s_{0} \in\right] 0, s_{-}\left[\right.$such that $2 \bar{\theta}\left(s_{0}\right)=\pi$.

Proof. Assume on the contrary, $\bar{\theta}(s) \in] \frac{\pi}{2}, \pi[$ for all $s \in] 0, s_{-}[$, and from (4.8)(4.14), we have that $\bar{x}^{\prime}<0, \bar{\theta}^{\prime}<0$ and $\bar{z}^{\prime}>0$ on $] 0, s_{-}[$. Hence, there exist

$$
\bar{x}_{-}=\lim _{s \rightarrow s_{-}} \bar{x}(s), \quad \bar{z}_{-}=\lim _{s \rightarrow s_{-}} \bar{z}(s), \quad \bar{\theta}_{-}=\lim _{s \rightarrow s_{-}} \bar{\theta}(s),
$$

and as $]-s_{-}, s_{+}$[ is the maximal interval of existence of $\gamma$, we have that either $\bar{x}_{-}=0$ or $\bar{z}_{-}=\infty$. Therefore, $\gamma^{-}$is the graph of a convex function $\bar{u}=\bar{u}(\bar{x})$ on $] \bar{x}_{-}, x_{0}\left[\right.$ such that either $\bar{x}_{-}=0$ or $\lim _{\bar{x} \rightarrow \bar{x}_{-}} \bar{u}(\bar{x})=+\infty$.

In the first case, if $\lim _{\bar{x} \rightarrow \bar{x}_{-}} \bar{u}(\bar{x})=+\infty$, from the convexity of $\bar{u}$ we get that $\bar{\theta}_{-}=\frac{\pi}{2}$ and there exists a sequence $\left\{s_{n}\right\} \rightarrow s_{-}$satisfying $\bar{\theta}^{\prime}\left(s_{n}\right) \rightarrow 0$, but then, from (4.8),

$$
\begin{aligned}
0 & =\lim _{n \rightarrow \infty} \bar{\theta}^{\prime}\left(s_{n}\right)=\lim _{n \rightarrow \infty} \cos \left(\bar{\theta}\left(s_{n}\right)\right) \dot{\varphi}\left(\bar{z}\left(s_{n}\right)\right)-\frac{\sin \left(\bar{\theta}\left(s_{n}\right)\right)}{\bar{x}\left(s_{n}\right)} \\
& \leq \lim _{n \rightarrow \infty} \cos \left(\bar{\theta}\left(s_{n}\right)\right) \dot{\varphi}\left(\bar{z}\left(s_{n}\right)\right) \leq 0 .
\end{aligned}
$$

Thus,

$$
0=\lim _{n \rightarrow \infty} \cos \left(\bar{\theta}\left(s_{n}\right)\right) \dot{\varphi}\left(\bar{z}\left(s_{n}\right)\right)=\lim _{n \rightarrow \infty} \frac{\sin \left(\bar{\theta}\left(s_{n}\right)\right)}{\bar{x}\left(s_{n}\right)}=\frac{1}{\bar{x}_{-}} \neq 0,
$$

which is a contradiction.

If $\bar{x}_{-}=0$ then, from [22, Theorem 2], $\lim _{\bar{x} \rightarrow 0} \bar{u}(\bar{x})=+\infty$ and arguing as above we also obtain a contradiction.

Lemma 4.8. If $s \in] s_{0}, s_{-}[$, then $0<2 \bar{\theta}(s)<\pi$.

Proof. It is clear because $\bar{\theta}^{\prime}<0$ on $\bar{\theta}^{-1}\left(\frac{\pi}{2}\right)$ and $\bar{\theta}^{\prime}>0$ on $\bar{\theta}^{-1}(0)$.

Lemma 4.9. $\bar{\theta}$ has a minimum at a point $\left.s_{1} \in\right] s_{0}, s_{-}\left[\right.$and $\bar{\theta}^{\prime}>0$ on $] s_{1}, s_{-}[$

Proof. Assume that $\bar{\theta}^{\prime}<0$ on $] s_{0}, s_{-}\left[\right.$. Then, from Lemma 4.8, $\bar{x} \nearrow \bar{x}_{-}$, $\bar{z} \nearrow \bar{z}_{-}$and $\bar{\theta} \searrow \bar{\theta}_{-} \in\left[0, \frac{\pi}{2}\left[\right.\right.$ when $s \rightarrow s_{-}$. In particular, there is a sequence $\left\{s_{n}\right\} \rightarrow s_{-}$satisfying $\lim _{n \rightarrow \infty} \bar{\theta}^{\prime}\left(s_{n}\right)=0$.

Under this assumption, we assert that $\bar{\theta}_{-} \neq 0$ and $\bar{x}_{-}<+\infty$, otherwise

$$
\begin{aligned}
0 & =\lim _{n \rightarrow \infty} \bar{\theta}^{\prime}\left(s_{n}\right)=\lim _{n \rightarrow \infty} \cos \left(\bar{\theta}\left(s_{n}\right)\right) \dot{\varphi}\left(\bar{z}\left(s_{n}\right)\right)-\frac{\sin \left(\bar{\theta}\left(s_{n}\right)\right)}{\bar{x}\left(s_{n}\right)} \\
& =\lim _{n \rightarrow \infty} \cos \left(\bar{\theta}_{-}\right) \lim _{n \rightarrow \infty} \dot{\varphi}\left(\bar{z}\left(s_{n}\right)\right) \geq \cos \left(\bar{\theta}_{-}\right) \dot{\varphi}\left(z_{0}\right)>0
\end{aligned}
$$


which is a contradiction. Thus, $\gamma^{-}$is the graph of a concave function $\bar{u}=\bar{u}(\bar{x})$ on a bounded interval $] \bar{x}\left(s_{0}\right), \bar{x}_{-}$[ satisfying $\lim _{\bar{x} \rightarrow \bar{x}_{-}} \bar{u}(\bar{x})=+\infty$ but this is also a contradiction because $\bar{\theta}$ is strictly decreasing on $] s_{0}, s_{-}[$.

Hence, there exists $\left.s_{1} \in\right] s_{0}, s_{-}\left[\right.$such that $\bar{\theta}^{\prime}\left(s_{1}\right)=0$. Moreover, from (4.8),

$$
\bar{\theta}^{\prime \prime}\left(s_{1}\right)=\sin \left(\bar{\theta}\left(s_{1}\right)\right) \cos \left(\bar{\theta}\left(s_{1}\right)\right)\left(\ddot{\varphi}\left(\bar{z}\left(s_{1}\right)\right)+\frac{1}{\bar{x}^{2}\left(s_{1}\right)}\right)>0,
$$

and $s_{1}$ is a local minimum of $\bar{\theta}$. Now, arguing as in Theorem 4.5, we can prove that, on the interval $] \bar{x}\left(s_{1}\right), \bar{x}_{-}\left[, \gamma^{-}\right.$is the graph of a convex function satisfying

$$
\lim _{\bar{x} \rightarrow \bar{x}_{-}} \bar{u}(\bar{x})=+\infty
$$

Lemma 4.10. The profile curve $\gamma$ is embedded.

Proof. Let $\left.s_{0} \in\right]-s_{-}, 0[$ the point given by the Lemma 4.7 and consider the following branches of $\gamma$ determined by $\left.\gamma\right|_{-s_{-}, s_{0}[}$ and $\left.\gamma\right|_{s_{0}, s_{+}[}$, respectively, parametrized by

$$
\begin{aligned}
& \left.u_{+}(x)=\left(x, u_{+}(x)\right) \text { for any } x \in\right] x_{0}, x\left(s_{-}\right)[ \\
& \left.u_{-}(s)=\left(x, u_{-}(x)\right) \text { for any } x \in\right] x_{0}, x\left(s_{+}\right)[,
\end{aligned}
$$

where $u$ is solution of the Eq. (4.12). Now, define the following smooth function $d(x)=u_{+}^{\prime}(x)-u_{-}^{\prime}(x)$. It is clear that $d(x)>0$ for $\left.x \in\right] x_{0}, x_{0}+\delta[$ for some $\delta>0$. Suppose that there exists a first $r \geq x_{0}+\delta$ such that $d(r)=0$ and $d^{\prime}(r) \leq 0$. Consequently, $u_{+}(x)>u_{-}(x)$ for any $\left.x \in\right] x_{0}, r[$ and from the equation 4.12 , we get to contradiction since,

$$
d^{\prime}(r)=\left(1+u^{\prime}(r)^{2}\right)\left(\dot{\varphi}\left(u_{+}(r)\right)-\dot{\varphi}\left(u_{-}(r)\right)\right)>0 .
$$

Thus, $d^{\prime}>0$ everywhere and integrating $u_{+}(x)>u_{-}(x)$ for any $x \geq x_{0}$.

Theorem 4.11. For every $x_{0}>0$, there exists a complete embedded rotational $\left[\varphi, \vec{e}_{3}\right]$-minimal, see Fig. 5 (right) with the annulus topology whose distance to axis of revolution is $x_{0}$ and whose generating curve $\gamma$ is of winglike type see Fig. 5 (left).

These examples will be called $\left[\varphi, \vec{e}_{3}\right]$-minimal catenoids.

Proof. It follows from Lemmas 4.7, 4.8, 4.9 and 4.10.

Proposition 4.12. Under the above conditions, the following statements hold:

1. If $\dot{\varphi}$ has at most a linear growth, then $\omega_{+}=+\infty$ and $\bar{x}_{-}=+\infty$.

2. If $\dot{\varphi}$ growths as $u^{\alpha}$ for some $\alpha>1$, then $\omega_{+}, \bar{x}_{-} \in \mathbb{R}$.

Proof. If $\dot{\varphi}$ has at most a linear growth, then there must be a constant $c>0$ such that $\dot{\varphi}(u) / u \leq c$ outside a compact set. Thus, from the equation (4.12), when $x$ is large enough the following inequalities hold:

$$
x \geq \frac{u^{\prime}}{\dot{\varphi}(u)}(x) \geq \frac{1}{c} \frac{u^{\prime}}{u}(x) .
$$



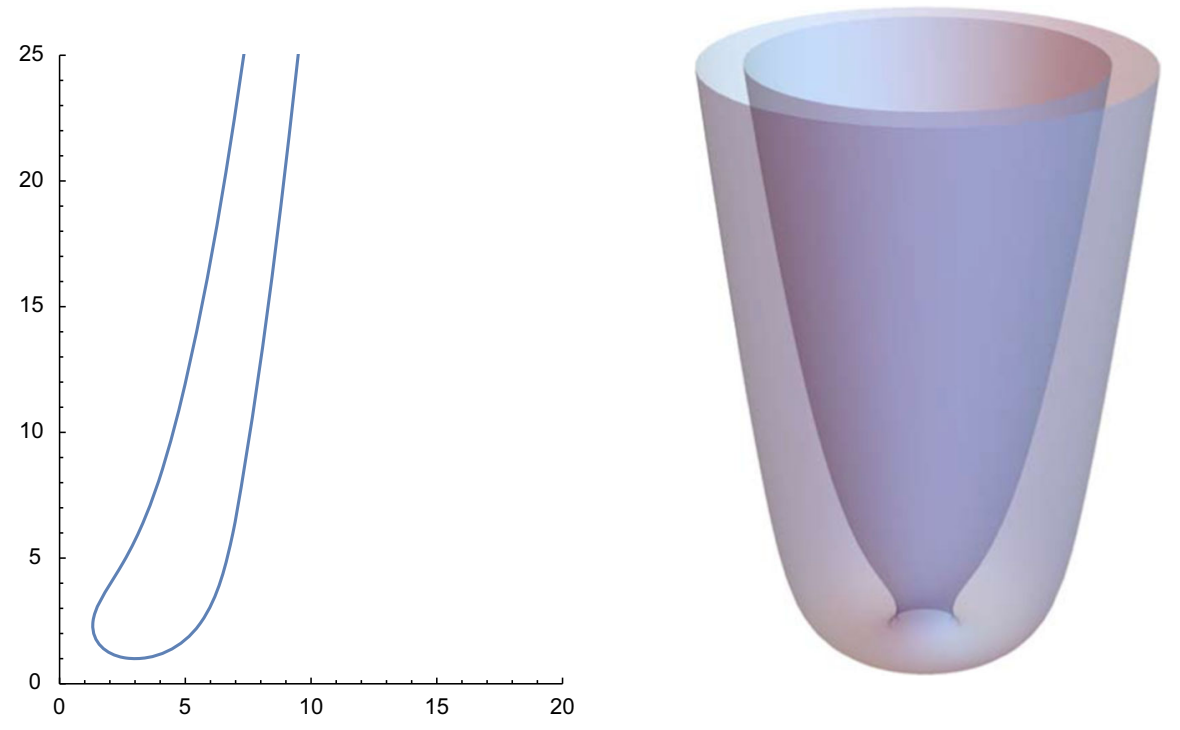

Figure 5. $\left[\varphi, \vec{e}_{3}\right]$-minimal catenoid with $\dot{\varphi}(u)=e^{-1 / u}$

Integrating both members of the inequality (4.15), we get that

$$
\frac{x^{2}}{2}-\frac{x_{0}^{2}}{2} \geq \frac{1}{c} \log \left(\frac{u(x)}{u\left(x_{0}\right)}\right) \text { for some } x_{0}>0 \text {. }
$$

Hence, $\omega_{+}=+\infty$ and $\bar{x}_{-}=+\infty$.

Let us go to consider now that

$$
\lim _{u \rightarrow+\infty} \frac{\dot{\varphi}(u)}{u^{\alpha}}=M \neq 0 \quad \text { for some } \alpha>1,
$$

and suppose that $\omega_{+}=+\infty$. Then, from the Theorems 4.5 and 4.11 , the real function $f$ given by

$$
f(r):=\frac{u^{\prime}(r)}{M u^{\alpha}(r)}
$$

has, for $r$ large enough, a bounded and strictly monotone primitive $F(u)(r)$. Hence, there exists a sequence $\left\{r_{n}\right\} \nearrow+\infty$ such that

$$
\lim _{n \rightarrow \infty} f\left(r_{n}\right)=0 \text {. }
$$

Claim 4.13. The function $f$ satisfies that $\lim _{r \rightarrow \infty} \frac{f(r)}{r}=0$.

Proof of Claim 4.13. Assuming on the contrary, there exists $\delta>0$ and a sequence $\left\{s_{n}\right\} \nearrow+\infty$ such that

$$
f\left(s_{n}\right)>\frac{f\left(s_{n}\right)}{s_{n}}>\delta,
$$

which together (4.17), says that $f^{-1}(\delta)$ is unbounded real subset containing a divergent sequence to $+\infty$. 
But, from the equation (4.1), the function $f$ satisfies the following differential equation:

$$
f^{\prime}=\left(\frac{\dot{\varphi}(u)}{M u^{\alpha}}-\frac{f(r)}{r}\right)+M^{2} f^{2} u^{2 \alpha}\left(\frac{\dot{\varphi}(u)}{M u^{\alpha}}-\frac{f}{r}-\frac{\alpha}{M} u^{-\alpha+1}\right)
$$

and we obtain that there exists $\hat{r} \in f^{-1}(\delta)$ such that $f^{\prime}(r)>1$ for any $r \in f^{-1}(\delta), r \geq \hat{r}$, which is impossible because $f^{-1}(\delta)$ is unbounded.

From (4.18), Claim 4.13 and using that $u$ diverges to $+\infty$ we get that, for $r$ sufficiently large, the following inequality holds:

$$
\frac{2 f^{\prime}}{1+f^{2}}>1
$$

By integration of this expression, we conclude that $\omega_{+}<+\infty$.

Remark 4.14. Notice that $\omega_{+}=+\infty$ does not imply that $\dot{\varphi}$ has at most a linear growth. For example, by taking $\dot{\varphi}(u)=u \log (u)$ with $u \geq 1$ and by the integration of both members in (4.15), we get that

$$
\frac{x^{2}}{2}-\frac{x_{0}^{2}}{2} \geq \log \left(\log \left(\frac{u(x)}{u\left(x_{0}\right)}\right)\right) \text { for some } x_{0}>0 \text {. }
$$

Thus, $\omega_{+}=+\infty$ but the function $\log (u)$ is not bounded.

\section{Asymptotic Behavior of Rotational Examples}

Clutterbuck, Schnürer and Schulze studied in [4] the asymptotic behavior of solitons rotationally symmetric. They proved that the problem

$$
\begin{cases}u^{\prime \prime}=\left(1+u^{\prime 2}\right)\left(1-\frac{u^{\prime}}{r}\right), & r>R, \\ u(R)=u_{0} \in \mathbb{R}, & u^{\prime}(R)=u_{1} \in \mathbb{R} .\end{cases}
$$

has a unique $\mathcal{C}^{\infty}$-solution $u$ on $[R, \infty[$. Moreover, as $r \rightarrow \infty, u$ has the following asymptotic expansion:

$$
u(r)=\frac{r^{2}}{2}-\log (r)+O\left(r^{-2}\right) .
$$

Due to the arbitrariness of the problem (4.1), it is impossible to find a general asymptotic behavior of their solutions because if you consider any strictly convex smooth function $u=u(r), r>R$, one can find a function $\varphi$ such that $u$ is a solution of (4.1).

Proposition 4.12 motivates to consider $\varphi:] a,+\infty[\longrightarrow \mathbb{R}$ a smooth function satisfying (4.9) and with a quadratic growth, that is, with the following asymptotic behavior:

$$
\lim _{u \rightarrow \infty} \ddot{\varphi}(u)=\alpha \geq 0 \quad \text { and } \quad \lim _{u \rightarrow \infty}(\dot{\varphi}(u)-\alpha u)=\beta \in \mathbb{R} .
$$

In this case, we are going to generalize the result in [4] to the following problem:

$$
\begin{cases}u^{\prime \prime}=\left(1+u^{\prime 2}\right)\left(\dot{\varphi}(u)-\frac{u^{\prime}}{r}\right), & r>r_{0} \geq 0, \\ u\left(r_{0}\right)=u_{0}>a, & u^{\prime}\left(r_{0}\right)=u_{1} \geq 0,\end{cases}
$$


with $\varphi:] a, \infty[\longrightarrow \mathbb{R}$ satisfying (4.9) and (5.2).

Remark 5.1. Observe that if $\alpha>0$, then $u$ is solution of (4.1) if and only if $v=u+\frac{\beta-\widetilde{\beta}}{\alpha}$ is solution of

$$
v^{\prime \prime}=\left(1+v^{\prime 2}\right)\left(\dot{\psi}(v)-\frac{v^{\prime}}{r}\right)
$$

where $\psi(v)=\varphi\left(v-\frac{\beta-\widetilde{\beta}}{\alpha}\right)$ satisfies

$$
\lim _{v \rightarrow \infty} \ddot{\psi}(v)=\alpha \geq 0 \quad \text { and } \quad \lim _{v \rightarrow \infty}(\dot{\psi}(v)-\alpha v)=\widetilde{\beta} .
$$

It is also clear that $\frac{v^{\prime}}{\dot{\psi}(v)}=\frac{u^{\prime}}{\dot{\varphi}(u)}$.

Theorem 5.2. (Case $\alpha>0)$ Assume that $\dot{\varphi}\left(u_{0}\right) r_{0} \geq u_{1}$ and $\alpha>0$. Then, the problem (5.3) has an unique strictly convex $\mathcal{C}^{\infty}$-solution $u$ on $\left[r_{0}, \infty[\right.$. Moreover, as $r \rightarrow \infty$, we have the following asymptotic expansion:

$$
\begin{aligned}
& \dot{\varphi}(u)(r)=e^{\frac{1}{2} \alpha r^{2}+o\left(r^{2}\right)} \\
& \frac{u^{\prime}}{\dot{\varphi}(u)}(r)=r-\alpha r \dot{\varphi}(u)^{-2}(r)+o\left(r \dot{\varphi}(u)^{-2}(r)\right),
\end{aligned}
$$

Proof. First of all, arguing as in Theorems 4.5, 4.11 and Proposition 4.12, (5.3) has a unique $\mathcal{C}^{\infty}$-solution $u$ on $\left[r_{0}, \infty\right.$ [ which is strictly convex function satisfying that $\lim _{r \rightarrow \infty} u(r)=\infty$. Hence, from (4.1),

$$
r \dot{\varphi}(u)>u^{\prime}, \quad r \geq r_{0} .
$$

From Remark 5.1, to study the asymptotic behavior of $\frac{u^{\prime}}{\dot{\varphi}(u)}$, it is not a restriction to assume that $\beta>0$.

Take $\epsilon>0$ such that $\beta>2 \varepsilon$, from (5.2) there exists $r_{\varepsilon}$ such that if $r \geq r_{\varepsilon}$,

$$
-\varepsilon<\dot{\varphi}(u)(r)-\alpha u(r)-\beta<\varepsilon, \quad-\varepsilon<\ddot{\varphi}(u)(r)-\alpha<\varepsilon .
$$

Lemma 5.3. Consider for any $R>r_{0}$, the function

$$
\zeta_{R}(r):=g_{\varepsilon}\left(u(R)+\int_{R}^{r} t \dot{\varphi}(u)(t) \mathrm{d} t\right), \quad r \geq R, \quad g_{\varepsilon}=\frac{\beta-2 \varepsilon}{\beta+\varepsilon} .
$$

Then, there exists $r_{1} \in \mathbb{R}$, depending only on $\varepsilon$, such that for any $R \geq r_{1}, \zeta_{R}$ satisfies the following inequality:

$$
\zeta_{R}^{\prime \prime}<\left(1+\zeta_{R}^{\prime 2}\right)\left(\dot{\varphi}\left(\zeta_{R}\right)-\frac{\zeta_{R}^{\prime}}{r}\right), \quad r \geq R .
$$

Proof. From the inequality (5.6), $\zeta_{R}(r)>u(r) g_{\varepsilon}$. Hence, from (5.7), when $r$ is large enough, we have

$$
\dot{\varphi}\left(\zeta_{R}\right)(r)>\alpha g_{\varepsilon} u(r)+\beta-\varepsilon .
$$

Using (5.6), (5.7) and by a straightforward computation,

$$
\zeta_{R}^{\prime \prime}(r)<g_{\varepsilon} \dot{\varphi}(u)(r)\left(1+(\alpha+\varepsilon) r^{2}\right), \quad r \geq r_{\varepsilon},
$$


On the other hand, from (5.9) and (5.7), when $r \geq r_{\varepsilon}$, the following inequality holds:

$$
\left(1+\zeta_{R}^{\prime 2}\right)\left(\dot{\varphi}\left(\zeta_{R}\right)-\frac{\zeta_{R}^{\prime}}{r}\right)>\varepsilon\left(1+\dot{\varphi}(u)^{2} r^{2} g_{\varepsilon}^{2}\right) .
$$

Thus, (5.8) follows from (5.9), (5.10), (5.11) bearing in mind that $u \rightarrow+\infty$ when $r \rightarrow+\infty$.

Lemma 5.4. For any $R \geq r_{0}$ there exists $r_{R} \geq R$ such that $u^{\prime}\left(r_{R}\right)-\zeta_{R}^{\prime}\left(r_{R}\right)>$ 0 .

Proof. Assuming on the contrary, if $u^{\prime}(r)-\zeta_{R}^{\prime}(r) \leq 0$ for any $r>R$, then the following inequalities holds:

$$
\frac{u^{\prime \prime}(r)}{1+u^{\prime^{2}}(r)} \geq \frac{3 \varepsilon}{\beta+\varepsilon} \dot{\varphi}(u)(r)>\frac{3 \varepsilon}{\beta+\varepsilon} \dot{\varphi}(u)\left(r_{0}\right),
$$

Integrating, we can find a finite radius $\bar{r}$ such that $u^{\prime} \rightarrow+\infty$ as $r \rightarrow \bar{r}$, getting a contraction since the solution $u$ is defined for all $r>r_{0}$.

Let us consider the function $d=u^{\prime}-\zeta_{R}^{\prime}$ on $[R, \infty[$. From Lemmas 5.3 and 5.4 , we can find $R \gg r_{0}$ verifying $u(R)>0, d(R)>0$ and such that the inequality (5.8) holds. Hence, if there exists a first $s \geq R$ such that $d(s)=0$ and $d^{\prime}(s)<0$, we have

$$
0>d^{\prime}(s)=\left(1+u^{\prime}(s)^{2}\right)\left(\dot{\varphi}(u(s))-\dot{\varphi}\left(\zeta_{R}(s)\right)\right) .
$$

On the other hand, as $d(r)>0$ for any $r \in] R, s$, we have by integration of d' that

$$
u(s)>\zeta_{R}(s)+u(R)-\zeta_{R}(R)=\zeta_{R}(s)+\frac{3 \varepsilon}{\beta+\varepsilon} u(R)>\zeta_{R}(s),
$$

and (4.9) gives that $d^{\prime}(s)>\dot{\varphi}(u(s))-\dot{\varphi}\left(\zeta_{R}(s)\right)>0$ which is a contradiction. Thus, $d(r)>0$ for $r$ large enough and using the inequality (5.6), we get

$$
\frac{u^{\prime}(r)}{\dot{\varphi}(u)(r)}=r+\mathcal{V}_{1}(r), \quad \text { with } \quad \lim _{r \rightarrow+\infty} \frac{\mathcal{V}_{1}(r)}{r}=0
$$

Moreover, from the previous formula (5.12) and L'Hôpital's rule, we also get that

$$
\lim _{r \rightarrow+\infty} \frac{\log \left(\dot{\varphi}^{2}(u(r))\right)}{\alpha r^{2}}=1
$$

and $\dot{\varphi}(u)$ has the following asymptotic expansion:

$$
\dot{\varphi}(u)(r)=e^{\frac{1}{2} \alpha r^{2}+o\left(r^{2}\right)} .
$$

Lemma 5.5. $\mathcal{V}_{1} \rightarrow 0$ as $r \rightarrow+\infty$.

Proof. As $\mathcal{V}_{1}$ is sublinear, we have that for $r$ large enough, $\left|\mathcal{V}_{1}(r)\right|<c r$ for all $c>0$. Moreover, from (5.3) and the inequality (5.6), $\mathcal{V}_{1}$ is a non-positive function and it satisfies the following differential equation:

$$
\mathcal{V}_{1}^{\prime}(r)=-\frac{\mathcal{V}_{1}(r)}{r}\left(1+\dot{\varphi}(u)^{2}(r)\left(r+\mathcal{V}_{1}(r)\right)^{2}\right)-1-\ddot{\varphi}(u)(r)\left(r+\mathcal{V}_{1}(r)\right)^{2}
$$


Take $\varepsilon>0$ and $R \gg r_{0}$. If $r \geq R$ and $\mathcal{V}_{1}(r) \leq-\varepsilon$, from the sublinearity, we can suppose that $-r / 2<\mathcal{V}_{1}(r)$, and

$$
\frac{r^{2}}{4}<\left(r+\mathcal{V}_{1}(r)\right)^{2}<(c+1)^{2} r^{2}
$$

Now, choosing $R$ large enough, the Eq. (5.14) and the inequalities (5.7) and (5.15) give

$$
\mathcal{V}_{1}^{\prime}(r) \geq-1+\frac{\varepsilon}{r}+r\left(\frac{\varepsilon}{4} \dot{\varphi}(u)^{2}(r)-(\alpha+\varepsilon)(c+1)^{2} r\right) .
$$

Using the conditions (4.9) and the asymptotic behavior (5.13), $R$ may be chosen large enough so that

$$
\dot{\varphi}(u)^{2}(r) \geq \frac{4}{\varepsilon}\left((\alpha+\varepsilon)(c+1)^{2} r+\frac{1}{r}\left(c+1-\frac{\varepsilon}{r}\right)\right), \quad r \geq R .
$$

Thus, if $R$ is large enough and $r \geq R$ where $\mathcal{V}_{1}(r) \leq-\varepsilon$, then $\mathcal{V}_{1}^{\prime}(r) \geq c>0$. Hence, $\mathcal{V}_{1}(r) \geq-\varepsilon$ for $r$ large enough and we conclude the proof.

Lemma 5.6. $\lim _{r \rightarrow+\infty} \frac{1}{r} \dot{\varphi}^{2}(u)(r) \mathcal{V}_{1}(r)=-\alpha$.

Proof. If $\lambda(r)=\frac{1}{r} \dot{\varphi}^{2}(u)(r) \mathcal{V}_{1}(r)$, then from (5.3) and (5.12), we have

$$
\begin{aligned}
\lambda^{\prime}(r)= & \dot{\varphi}^{2}(u)(r)\left(2 \mathcal{V}_{1}(r)\left(\ddot{\varphi}(u)(r)\left(1+\frac{\mathcal{V}_{1}(r)}{r}\right)-\frac{1}{r^{2}}\right)-\frac{1}{r}\right) \\
& +\dot{\varphi}^{2}(u)(r)\left(\frac{\left(r+\mathcal{V}_{1}(r)\right)^{2}}{r}(-\ddot{\varphi}(u)(r)-\lambda(r))\right) .
\end{aligned}
$$

Fix $\varepsilon>0$ and $R$ large enough. Consider points $r \geq R$ where $\lambda(r) \geq-\alpha+\epsilon$, then

$$
-\ddot{\varphi}(u)(r)-\lambda(r) \leq-\ddot{\varphi}(u)(r)+\alpha-\varepsilon
$$

and if $R$ is large enough, from (5.2) and (5.17), we also get that

$$
-\ddot{\varphi}(u)(r)-\lambda(r) \leq-\frac{\varepsilon \alpha}{2}<0
$$

and then $\lambda^{\prime}(r)<-1$ when $R$ is chosen sufficiently large. Hence, we obtain that $\lambda(r) \leq-\alpha+\varepsilon$ for $r$ large enough.

In a similar way, we may prove that $\lambda(r) \leq-\alpha-\varepsilon$ for $r$ sufficiently large.

Now, (5.5) follows from (5.12), (5.13) and Lemmas 5.5 and 5.6.

Theorem 5.7. (Case $\alpha=0$ ) Assume that $\dot{\varphi}\left(u_{0}\right) r_{0} \geq u_{1}, \alpha=0$ and $\beta>$ 0 . Then, the problem (5.3) has an unique strictly convex $\mathcal{C}^{\infty}$-solution $u$ on $\left[r_{0}, \infty[\right.$. Moreover, if

$$
\lim _{u \rightarrow+\infty} u \ddot{\varphi}(u)=0,
$$

we have the following asymptotic expansion:

$$
\frac{u^{\prime}}{\dot{\varphi}(u)}(r)=r-\frac{1}{\beta^{2} r}+o\left(r^{-1}\right),
$$


Proof. Arguing as in Theorems 4.5, 4.11 and Proposition 4.12, (5.3) has a unique $\mathcal{C}^{\infty}$-solution $u$ on $\left[r_{0}, \infty[\right.$ which is strictly convex function satisfying that $\lim _{r \rightarrow \infty} u(r)=\infty$. Moreover, as Lemmas 5.3 and 5.4 also work in this case, we have the following asymptotic expansion:

$$
\frac{u^{\prime}}{\dot{\varphi}(u)}(r)=r+\mathcal{V}_{1}(r)
$$

where $\mathcal{V}_{1}$ verifies the same differential equation (5.14), is also non-positive and $\mathcal{V}_{1}(r) \rightarrow 0$. Moreover, from (5.2), $\dot{\varphi}$ writes as

$$
\dot{\varphi}(u)(r)=\beta+o(1) .
$$

Consider now the new function $\mathcal{V}_{2}(r)=r \dot{\varphi}^{2}(u)(r) \mathcal{V}_{1}(r)$. Then,

$$
\mathcal{V}_{2}^{\prime}=r \dot{\varphi}^{2}\left(2 \ddot{\varphi} \mathcal{V}_{1}\left(r+\mathcal{V}_{1}\right)-1+\frac{\left(r+\mathcal{V}_{1}\right)^{2}}{r^{2}}\left(-r^{2} \ddot{\varphi}-\mathcal{V}_{2}\right)\right) \text {. }
$$

From the expressions (5.18), (5.20) and L'Hôpital's rule, we have

$$
\lim _{r \rightarrow+\infty} \ddot{\varphi}(u(r)) r=0 \text { and } \lim _{r \rightarrow+\infty} \ddot{\varphi}(u(r)) r^{2}=0,
$$

and working as in Lemma 5.6 we can prove that $\mathcal{V}_{2}(r) \rightarrow-1$. Finally, the Theorem follows from the expansion (5.20) as $r \rightarrow+\infty$.

\subsection{Proof of Theorem A}

If $\alpha>0$, from (5.12) and (5.13), we can write

$$
\log (\dot{\varphi}(u))(r)=\frac{\alpha r^{2}}{2}+\Upsilon(r),
$$

where $\Upsilon^{\prime}=(\ddot{\varphi}-\alpha) r+\ddot{\varphi} \mathcal{V}_{1}$. Hence, as the first non-vanishing $a_{k}$ is positive, for $r$ large enough $\Upsilon$ is a decreasing function in $r$ such that $-\infty<c=$ $\lim _{r \rightarrow+\infty} \Upsilon(r)$ otherwise from Lemma 5.6, (1.10), (5.23) and using L'Hôpital's rule, we have that

$$
\begin{aligned}
+\infty & =\lim _{r \rightarrow+\infty} \dot{\varphi}^{2}(u)(r)=\lim _{r \rightarrow+\infty} \frac{e^{2 \Upsilon}}{e^{-\alpha r^{2}}}=\lim _{r \rightarrow+\infty} \frac{\left(e^{2 \Upsilon}\right)^{\prime}}{\left(e^{-\alpha r^{2}}\right)^{\prime}} \\
& =-\lim _{r \rightarrow+\infty} \frac{\dot{\varphi}(u)^{2}(r)\left((\ddot{\varphi}(u)(r)-\alpha) r+\ddot{\varphi}(u)(r) \mathcal{V}_{1}(r)\right)}{\alpha r}=\alpha a_{1},
\end{aligned}
$$

which is a contradiction.

Applying again L'Hôpital's rule to $\lim _{r \rightarrow+\infty} \frac{e^{2 \Upsilon}-e^{2 c}}{e^{-\alpha r^{2}}}$, we have

$$
\dot{\varphi}^{2}(u)(r)=e^{\alpha r^{2}+2 c}+O(1) \text { and } \lim _{r \rightarrow+\infty} O(1)=\alpha a_{1} .
$$

Thus, from Lemma 5.6 and Theorem 5.2,

$$
\varphi(u)^{\prime}(r)=r e^{\alpha r^{2}+2 c}+\alpha a_{1} r+o(r),
$$

and (1.11) follows by integration of the above expression.

If $\alpha=0$ then, the condition (5.18) follows from (5.20) and we have that

$$
\frac{u^{\prime}}{\dot{\varphi}(u)}(r)=r-\frac{1}{\beta^{2} r}+o\left(r^{-1}\right) \text {. }
$$


Now, by taking $\mathcal{V}_{3}(r)=\left(\mathcal{V}_{2}(r)+1\right) r^{2}$, we get

$$
\begin{aligned}
\mathcal{V}_{3}^{\prime}= & \frac{2 \mathcal{V}_{3}}{r}+r^{3} \dot{\varphi}^{2}\left(2 \ddot{\varphi} \mathcal{V}_{1}\left(r+\mathcal{V}_{1}\right)-1+\frac{\left(r+\mathcal{V}_{1}\right)^{2}}{r^{2}}\left(-r^{2} \ddot{\varphi}+1-\frac{\mathcal{V}_{3}}{r^{2}}\right)\right) \\
= & r \dot{\varphi}^{2}\left(\frac{2 \mathcal{V}_{3}}{\dot{\varphi}^{2} r^{2}}+2 r^{4} \ddot{\varphi} \frac{\mathcal{V}_{1}\left(r+\mathcal{V}_{1}\right)}{r^{2}}-r^{2}+\frac{\left(r+\mathcal{V}_{1}\right)^{2}}{r^{2}}\left(-r^{4} \ddot{\varphi}+r^{2}-\mathcal{V}_{3}\right)\right) \\
= & r \dot{\varphi}^{2} \frac{\left(r+\mathcal{V}_{1}\right)^{2}}{r^{2}}\left(-r^{4} \ddot{\varphi}+r^{2}\left(1-\frac{r^{2}}{\left(r+\mathcal{V}_{1}\right)^{2}}\right)-\mathcal{V}_{3}\right) \\
& +r \dot{\varphi}^{2}\left(\frac{2 \mathcal{V}_{3}}{\dot{\varphi}^{2} r^{2}}+2 r^{4} \ddot{\varphi} \frac{\mathcal{V}_{1}\left(r+\mathcal{V}_{1}\right)}{r^{2}}\right) .
\end{aligned}
$$

But, from (5.20) and L'Hôpital's rule, we obtain

$$
\begin{aligned}
& \lim _{r \rightarrow+\infty} \ddot{\varphi}(u(r)) r^{4}=-\frac{4 a_{1}}{\beta^{2}}, \\
& \lim _{r \rightarrow+\infty} r^{2}\left(1-\frac{r^{2}}{\left(r+\mathcal{V}_{1}\right)^{2}}\right)=-\frac{2}{\beta^{2}}
\end{aligned}
$$

thus, by working as in Lemma 5.6, we prove that

$$
\lim _{r \rightarrow \infty} \mathcal{V}_{3}(r)=\frac{-2+4 a_{1}}{\beta^{2}}
$$

Hence,

$$
\frac{u^{\prime}}{\dot{\varphi}(u)}(r)=r-\frac{1}{\beta^{2} r}-\frac{2-4 a_{1}}{\beta^{4} r^{3}}+o\left(r^{-3}\right),
$$

and (1.12) follows from integration in the above expression.

\section{Uniqueness of Globally Convex Solutions}

Along this section $\varphi:] a,+\infty[\longrightarrow \mathbb{R}$ will be a regular function satisfying the expansion (1.10).

For any $\theta \in\left[0,2 \pi\left[\right.\right.$, we consider $\vec{v}=(\cos \theta, \sin \theta, 0)$ and denote by $\Pi_{\vec{v}}(t)$ the vertical plane

$$
\Pi_{\vec{v}}(t)=\left\{p \in \mathbb{R}^{3} \mid\langle p, \vec{v}\rangle=t\right\}
$$

Definition 6.1. Let $\Sigma_{1}$ and $\Sigma_{2}$ be two arbitrary subsets of $\mathbb{R}^{3}$. We say that $\Sigma_{1}$ is on the right hand side of $\Sigma_{2}$ respect to $\Pi_{\vec{v}}(t)$ and write $\Sigma_{1} \geq_{\vec{v}} \Sigma_{2}$ if and only if for every point $q \in \Pi_{\vec{v}}(t)$ such that

$$
\pi^{-1}(q) \cap \Sigma_{1} \neq \emptyset \text { and } \pi^{-1}(q) \cap \Sigma_{2} \neq \emptyset,
$$

we have the following inequality:

$$
\inf \left\{\langle p, \vec{v}\rangle: p \in \pi^{-1}(q) \cap \Sigma_{1}\right\} \geq \sup \left\{\langle p, \vec{v}\rangle: p \in \pi^{-1}(q) \cap \Sigma_{2}\right\},
$$

where $\pi: \mathbb{R}^{3} \rightarrow \Pi_{\vec{v}}(t)$ denotes the orthogonal projection on $\Pi_{\vec{v}}(t)$. 
For an arbitrary subset $M$ of $\mathbb{R}^{3}$, we also consider the following subsets:

$$
\begin{aligned}
& \Sigma_{+}(t):=\{p \in M:\langle p, \vec{v}\rangle \geq t\} . \\
& \Sigma_{-}(t):=\{p \in M:\langle p, \vec{v}\rangle \leq t\} . \\
& \Sigma_{+}^{*}(t):=\left\{p+2(t-\langle p, \vec{v}\rangle) \vec{v} \in \mathbb{R}^{3}: p \in \Sigma_{+}(t)\right\} . \\
& \Sigma_{-}^{*}(t):=\left\{p+2(t-\langle p, \vec{v}\rangle) \vec{v} \in \mathbb{R}^{3}: p \in \Sigma_{-}(t)\right\} .
\end{aligned}
$$

From Theorem A, it is natural to study $\left[\varphi, \vec{e}_{3}\right]$-minimal surfaces whose behavior at infinity is of rotational type. To be more precise,

Definition 6.2. We say that a $\left[\varphi, \vec{e}_{3}\right]$-minimal end $\Sigma$ is smoothly asymptotic to a rotational-type example if $\Sigma$ can be expressed outside a ball as a vertical graph of a function $u_{\Sigma}$ so that, according to $\alpha$ is either positive or zero, one of the following expressions holds:

$$
\varphi\left(u_{\Sigma}\right)(x)=C e^{\alpha|x|^{2}}+O\left(|x|^{2}\right), \quad \text { if } \quad \alpha>0,
$$

where $C$ is a positive constant or up to a constant,

$$
\mathcal{G}\left(u_{\Sigma}\right)(x)=\frac{|x|^{2}}{2}-\frac{1}{\beta^{2}} \log (|x|)+O\left(|x|^{-2}\right),
$$

if $\alpha=0$ and $\beta>0$.

Let $\Sigma$ be an embedded $\left[\varphi, \vec{e}_{3}\right]$-minimal surface $\Sigma$ with a single end smoothly asymptotic to a bowl-type example. Then, there exists $R>0$ large enough such that $\Sigma \cap\left(\mathbb{R}^{3} \backslash B(0, R)\right)$ is the vertical graph of a function $u_{\Sigma}$ verifying either (6.2) if $\alpha>0$ or (6.3) if $\alpha=0$ and $\beta>0$.

Lemma 6.3. There exists $r_{1}>R$ such that if $t>r_{1}$ then $\Sigma_{+}(t)$ is a graph over $\Pi_{\vec{v}}(t)$.

Proof. It is clear that when $t>R, \Sigma_{+}(t)$ has only one component which is unbounded. Moreover, if $\alpha>0$ then from (6.2),

$$
\dot{\varphi}\left(u_{\Sigma}\right)(x)\left(d u_{\Sigma}\right)_{x}(\vec{v}) \geq 2 \alpha e^{\alpha|x|^{2}}\langle x, \vec{v}\rangle\left(C+e^{-\alpha|x|^{2}} g(|x|)\right),
$$

where

$$
\lim _{|x| \rightarrow \mid} \frac{g(|x|)}{|x|^{2}}=0 .
$$

Hence, there exists $r_{1}$ large enough such that if $\langle x, \vec{v}\rangle \geq r_{1}$, then $\left.\left(d u_{\Sigma}\right)_{x}(\vec{v})\right\rangle$ 0 , and in this case, the Lemma follows because $\Sigma$ is embedded and $\Sigma_{+}\left(r_{1}\right) \cup$ $\pi\left(\Sigma_{+}\left(r_{1}\right)\right)$ bounds a domain in $\mathbb{R}^{3}$.

When $\alpha=0$, a similar argument with (6.3) also works.

From Lemma 6.3, fixed $t>r_{1}, \Sigma_{+}^{*}(t) \cap\left\{p \in \mathbb{R}^{3}:\left\langle p, \vec{e}_{3}\right\rangle>R\right\}$ is the vertical graph of the function satisfying

$$
u_{t}^{*}(x)=u_{\Sigma}(x+2(t-\langle x, \vec{v}\rangle) \vec{v})
$$

Lemma 6.4. Consider $a>0$ not depending on $R$ and $\epsilon_{0}>0$. Then, for $R$ large enough and $t>a+\langle x, \vec{v}\rangle$, we have

$$
u_{t}^{*}(x)-u_{\Sigma}(x)>\epsilon_{0}>0 .
$$


Proof. If $\alpha>0$ then, from (6.2) and (6.4), we obtain

$$
\begin{aligned}
\varphi\left(u_{t}^{*}\right)(x)-\varphi\left(u_{\Sigma}\right)(x) \geq & C e^{\alpha|x|^{2}}\left(e^{4 \alpha t(t-\langle x, \vec{v}\rangle)}-1\right) \\
& -M\left(2|x|^{2}+4 t(t-\langle x, \vec{v}\rangle)\right),
\end{aligned}
$$

for some positive constant $M$. Hence, taking $\lambda$ such that

$$
\frac{1+\sqrt{1+\lambda}}{\lambda}<\frac{R}{2 t}
$$

and $R>\alpha^{-1}$, we have that $4 t(t-\langle x, \vec{v}\rangle) \leq \lambda|x|^{2}$ and

$$
\varphi\left(u_{t}^{*}\right)(x)-\varphi\left(u_{\Sigma}\right)(x)>C e^{\alpha R^{2}}\left(e^{4 \alpha R a}-1-M e^{-\alpha R^{2}}(\lambda+2) R^{2}\right)>0
$$

for $R$ large enough. The result follows because $\varphi$ is strictly increasing.

When $\alpha=0$, we can estimate $\mathcal{G}\left(u_{t}^{*}\right)(x)-\mathcal{G}\left(u_{\Sigma}\right)(x)$ as in [17, Claim 1, Step 3] and to use that $\mathcal{G}$ is a strictly increasing function.

\subsection{Proof of Theorem B}

The main idea is to use the Alexandrov's reflection principle, [1], for proving that $\Sigma$ is symmetrical with respect to $\Pi_{\vec{v}}(0)$. For proving that, it is not difficult to see that Lemmas 6.3 and 6.4 are the fundamental facts we need to check that all the steps in the proof of Theorem A in [17] can be adapted to our case and for getting to prove that $0 \in \mathcal{A}$ were

$$
\mathcal{A}:=\left\{t \geq 0: \Sigma_{+}(t) \text { is a graph over } \Pi_{\vec{v}}(t) \text { and } \Sigma_{+}^{*}(t) \geq_{\vec{v}} \Sigma_{-}(t)\right\} .
$$

A symmetrical argument gives that $\Sigma_{-}^{*}(0) \leq_{\vec{v}} \Sigma_{+}(0)$. Hence, $\Sigma_{+}^{*}(0)=\Sigma_{-}(0)$ and $\Sigma$ is symmetric respect to the plane $\Pi_{\vec{v}}(0)$. As $\vec{v}=(\cos \theta, \sin \theta, 0)$ represents any unit horizontal vector, $\Sigma$ would be a revolution surface touching the axis of revolution, that is, a $\left[\varphi, \vec{e}_{3}\right]$-minimal bowl.

Remark 6.5 . It would be interesting to give a similar results for $\left[\varphi, \vec{e}_{3}\right]$-maximal surfaces in the Lorentz-Minkowski space $\mathbb{L}^{3}$ using the Calabi's Type correspondence of [18].

\section{Acknowledgements}

The authors are grateful to Margarita Arias, José Antonio Gálvez and Francisco Martín for helpful comments during the preparation of this manuscript.

Funding Funding for open access charge: Universidad de Granada / CBUA.

Open Access. This article is licensed under a Creative Commons Attribution 4.0 International License, which permits use, sharing, adaptation, distribution and reproduction in any medium or format, as long as you give appropriate credit to the original author(s) and the source, provide a link to the Creative Commons licence, and indicate if changes were made. The images or other third party material in this article are included in the article's Creative Commons licence, unless indicated otherwise in a credit line to the material. If material is not included in the article's Creative Commons licence and your intended use is not permitted by 
statutory regulation or exceeds the permitted use, you will need to obtain permission directly from the copyright holder. To view a copy of this licence, visit http:// creativecommons.org/licenses/by/4.0/.

Publisher's Note Springer Nature remains neutral with regard to jurisdictional claims in published maps and institutional affiliations.

\section{References}

[1] Alexandrov, A.D.: Uniqueness theorems for surfaces in the large. Vestnik. Leninger. Univ. Math. 11, 5-17 (1956)

[2] Böme, R., Hildebrant, S., Tausch, E.: The two-dimensional analogue of the catenary. Pac. J. Math. 88(2), 247-278 (1980)

[3] Cheng, X., Mejia, T., Zhou, D.: Stability and compactness for complete $f$ minimal surfaces. Trans. Am. Math. Soc. 367(6), 4041-4059 (2015)

[4] Clutterbuck, J., Schnüre, O., Schulze, F.: Stability of translating solutions to mean curvature flow. Calc. Var. 29, 281-293 (2007)

[5] Dierkes, U.: Singular minimal surfaces. In: Hildebrandt, S. et al. (eds.) Geometric Analysis and Nonlinear Partial Differential Equations, pp. 177-193 (2003)

[6] Dierkes, U., Huisken, G.: The N-dimensional analogue of the catenary: Prescribed area. In: Jost, J. (ed.) Calculus ofVariations and Geometric Analysis, pp. 1-13. International Press (1996)

[7] Eschenburg, J.-H.: Maximum principle for hypersurfaces. Manuscr. Math. 64, 55-75 (1989)

[8] Gama, E.S., Heinonen, E., de Lira, H.J., Martin, F: Jenkins-Serrin problem for horizontal translating graphs in MimesmathbbR. arXiv:1901.07224v1

[9] Hildebrant, S.: On the regularity of solutions of two-dimensional variational problems with obstructions. Commun. Pure Appl. Math. 25, 479-496 (1972)

[10] Hildebrant, S.: Interior $\mathcal{C}^{1+\alpha}$-regularity of solutions of two-dimensional variational problems with obstacles. Math. Z. 131, 233-240 (1973)

[11] Hildebrant, S., Kaul, H.: Two-dimensional variational problems with obstructions, and Plateau's problem for H-surfaces in a Riemannian manifold. Commun. Pure Appl. Math. 25, 187-223 (1972)

[12] Hoffman, D., Martín, F., White, B.: Scherk-like translators for mean curvature flow. Preprint (2019). arXiv:1903.04617

[13] Hoffman, D., Ilmanen, T., Martín, F., White, B.: Graphical translators for mean curvature flow. Calc. Var. PDE's 58, Art. 117 (2019)

[14] Hoffman, D., Ilmanen, T., Martín, F., White, B.: Notes on translating solitons of the mean curvature flow. Preprint (2019). arXiv:1901.09101

[15] López, R.: Invariant singular minimal surfaces. Ann. Glob. Anal. Geom. 53, 521-541 (2018)

[16] Martín, F., Pérez-García, J., Savas-Halilaj, A., Smoczyk, K.: A characterization of the grim reaper cylinder. J. Reine Angew. Math. 2019(746), 209-234 (2016)

[17] Martín, F., Savas-Halilaj, A., Smoczyk, K.: On the topology of translating solitons of the mean curvature flow. Calc. Var. 54, 2853-2882 (2015)

[18] Martínez, A., Martínez Triviño, A.L.: A Calabi's type correspondence nonlinear analysis. Nonlinear Anal. 191 (2020). https://doi.org/10.1016/j.na.2019.111637 
[19] Nitsche, J.C.C.: A nonexistence theorem for the two-dimensional analogue of the catenary. Analysis 6, 143-156 (1986)

[20] Poisson, S.D.: Sur les surfaces elastique. Men. CL. Sci. Math. Phys. Inst. Frace, deux, pp. 167-225 (1975)

[21] Savas-Halilaj, A., Smoczyk, K.: Berstein theorems for length and area decreasing minimal maps. Calc. Var. 50, 549-577 (2014)

[22] Serrin, J.: Removable singularities of solutions of elliptic equations. II. Arch. Ration. Mech. Anal. 20, 163-169 (1965)

[23] Spruck, J., Xiao, L.: Complete translating solitons to the mean curvature flow in $\mathbb{R}^{3}$. arXiv:1703.01003v3

[24] Tausch, E.: A class of variational problems with linear growth. Math. Z 164, 159-178 (1978)

[25] Wang, X.J.: Convex solutions to the mean curvature flow. Ann. Math. 173, 1185-1239 (2011)

Antonio Martínez and A. L. Martínez-Triviño

Department of Geometry and Topology

University of Granada

18071 Granada

Spain

e-mail: amartine@ugr.es, aluismartinez@ugr.es

Received: November 11, 2020.

Accepted: September 27, 2021. 